\title{
8A Conceptual Model for Death Education
}

\section{Mahboubeh Dadfar ${ }^{*}$, David Lester ${ }^{2}$, Ali Asghar Asgharnejad Farid ${ }^{3}$, Mohammad Kazem Atef Vahid ${ }^{4}$, Behrooz Birashk $^{5}$}

${ }^{1}$ Department of Clinical Psychology, School of Behavioral Sciences and Mental Health-Tehran Institue of Psychiatry, International Campus, Iran University of Medical Sciences, Tehran, Iran

${ }^{2}$ Department of Psychology, Stockton University, New Jersey, USA

${ }^{3}$ Department of Clinical Psychology and Mental Health, School of Behavioral Sciences and Mental Health-Tehran Institue of Psychiatry, Iran University of Medical Sciences, Tehran, Iran

${ }^{4}$ Department of Clinical Psychology and Health Psychology, School of Behavioral Sciences and Mental Health-Tehran Institue of Psychiatry, Iran University of Medical Sciences, Tehran, Iran

${ }^{5}$ Department of Clinical Psychology, School of Behavioral Sciences and Mental Health-Tehran Institue of Psychiatry, Iran University of Medical Sciences, Tehran, Iran

\section{Article Info:}

Received: 25 Jul 2016

Revised: 12 Aug 2016

Accepted: 17 Oct 2016

\section{A BSTRACT}

Introduction: Death is inevitable reality and each person may have a unique attitude and response toward it. Death education refers to a variety of educational activities and experiences related to death. Its main issues are meanings and attitudes towards death, the process of dying, grief and bereavement, and the care of people who have been affected. One of the most important tasks in the end of life care is preparation for death. Despite the importance of planning for the end of life care, some of health care professionals have not enough knowledge and skills to prepare their clients for exposure to death. Death education is a relevant international approach to grief, bereavement, and suicide counseling and psychotherapy. There are various approaches to death education program, including deductive, experiential approaches, and 8A conceptual model. Death education program has formal and informal forms with different methods. Conclusion: The 8A model has been presented as a framework to provide death education to health care professionals, such as social workers, nurses, physicians, thanatologists, psychologists, and psychiatrists. The 8A model, via improving of awareness, knowledge, attitude and action in individuals, can change behaviors associated with death.

Key words:

1. Death

2. Mental Health

3. Education

*Corresponding Author: Mahboubeh Dadfar

E-mail:mahboubehdadfar@yahoo.com 


\section{مدل مفهومى 8A براى آموزش مرى}

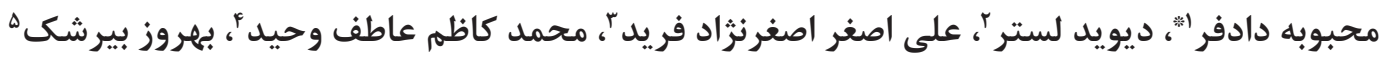

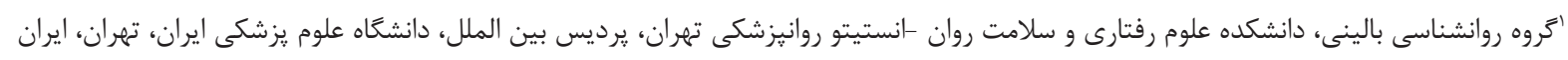

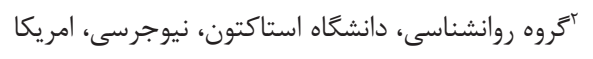

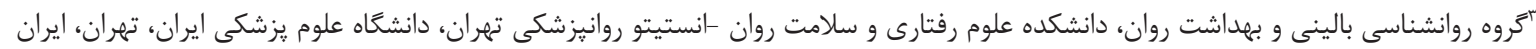

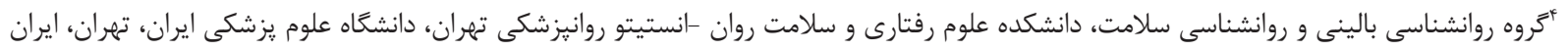

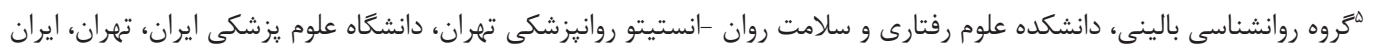

\section{اطلاعات مقاله:}

تاريخ بذيرش: צr مهر هوسا

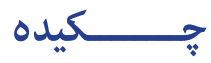

مقدمه: مرى واقعيت اجتنابنايذير است و هر شخصى ممكن است پاسخ و رفتارى منحصر به فرد نسبت

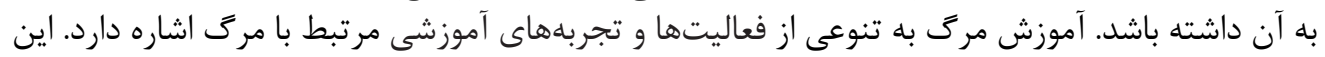

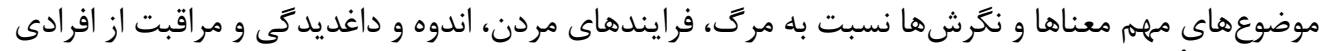

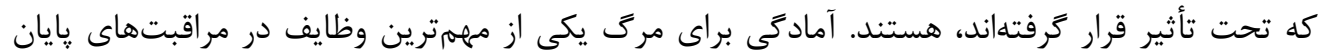

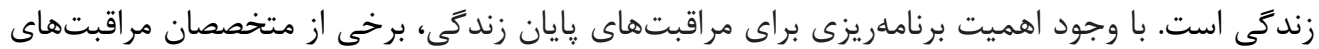

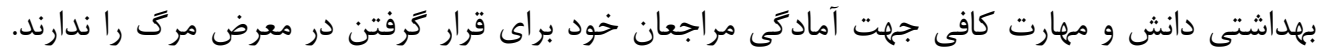

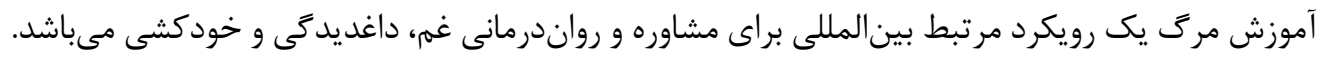

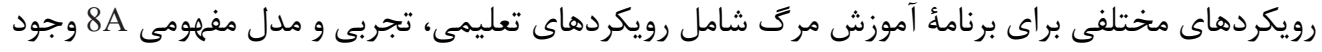

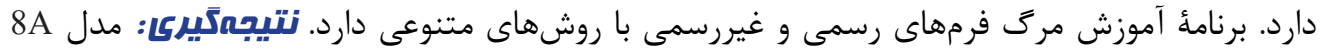

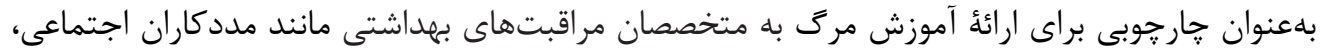

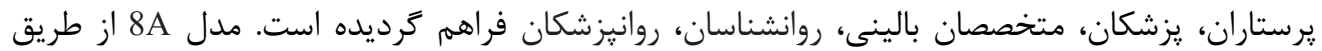

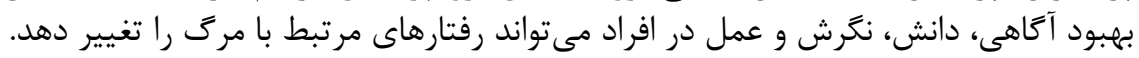

كليد وازهها: Fر r. بهداشت روان r. 


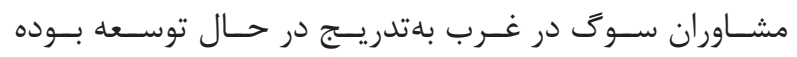

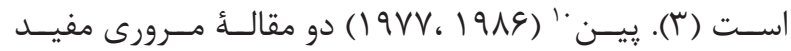

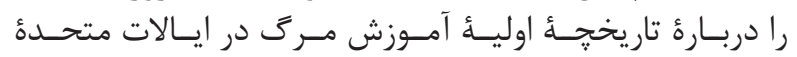

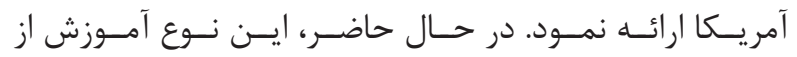

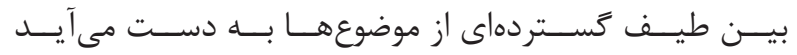

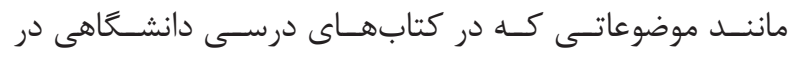

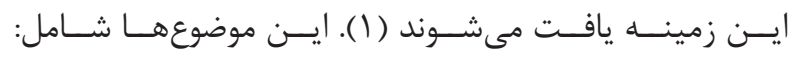

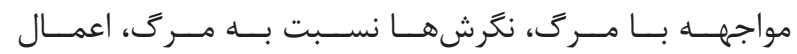

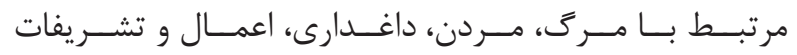

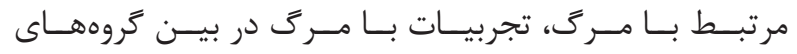

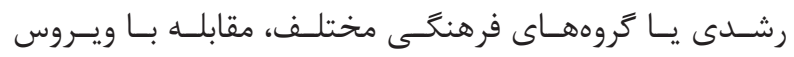

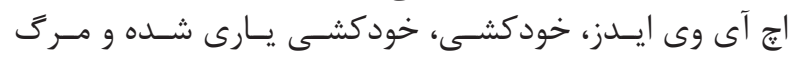

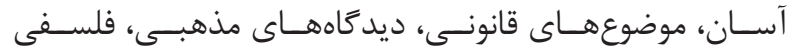

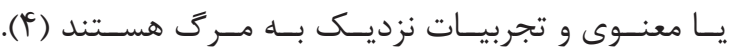

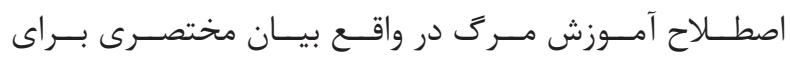

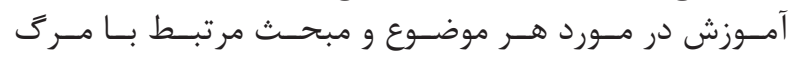

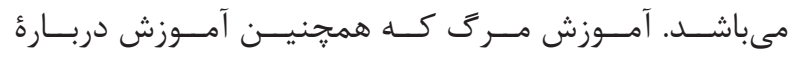

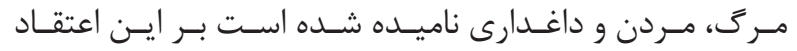

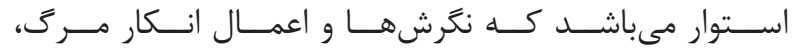

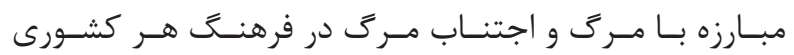

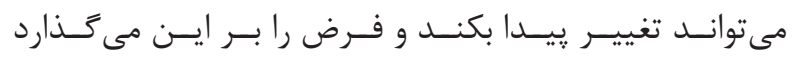

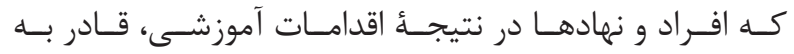

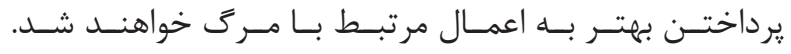

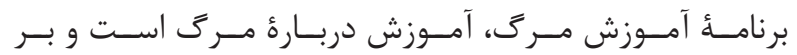

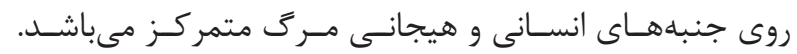

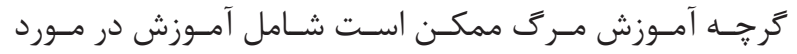

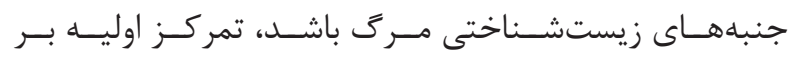

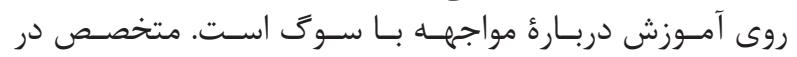

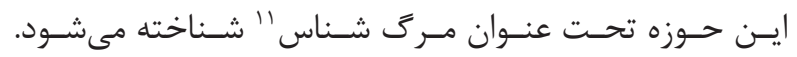

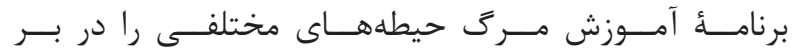

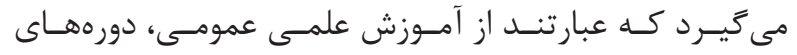

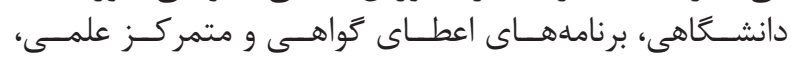

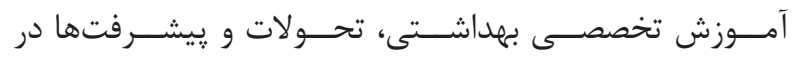

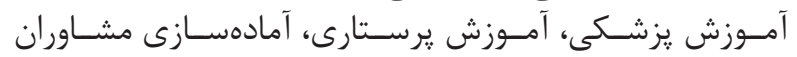

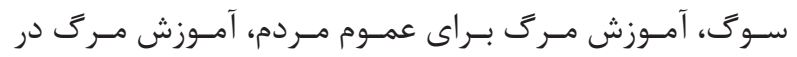

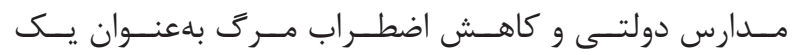

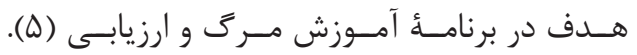

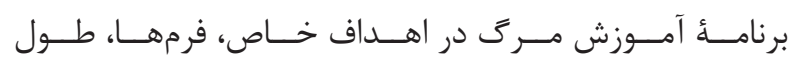

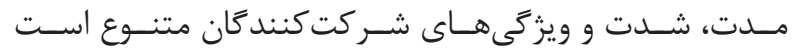

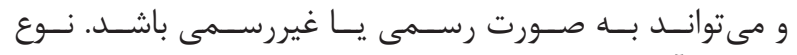

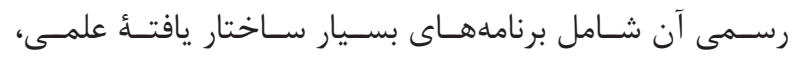

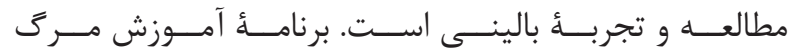

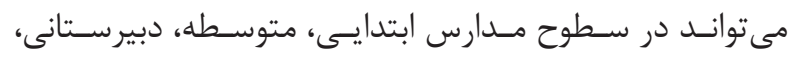

${ }^{1}$ Death anxiety

${ }^{2}$ Death concern

${ }^{3}$ Death fear

${ }^{4}$ Death obsession

${ }^{5}$ Death dpression

${ }^{6}$ Contemplators
مقدمه - مق

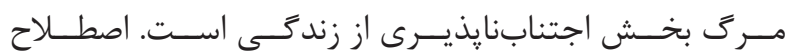

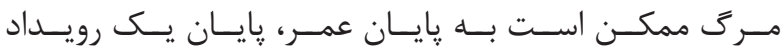

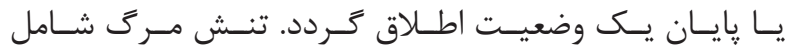

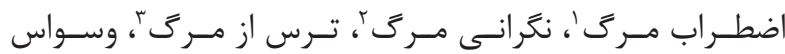

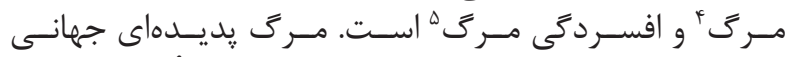

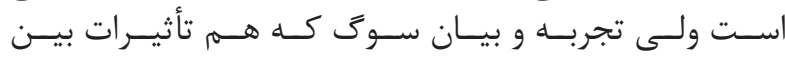

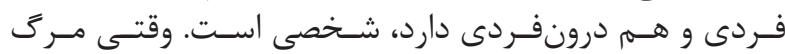

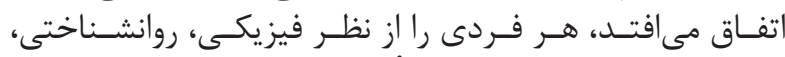

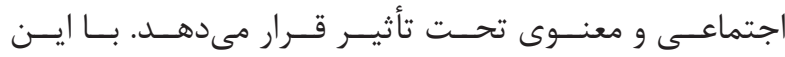

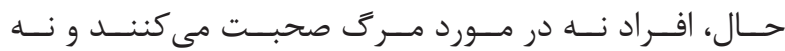

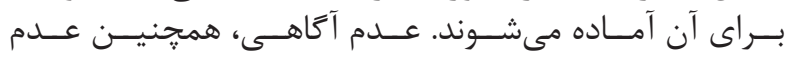

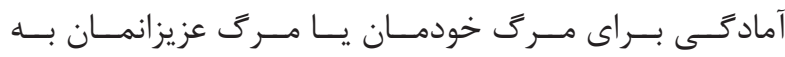

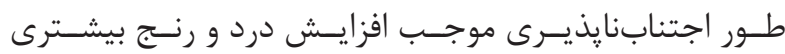

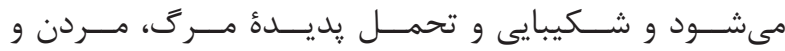

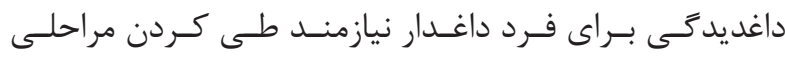

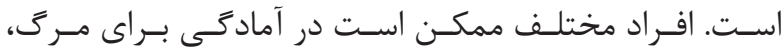

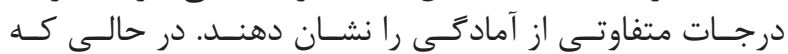

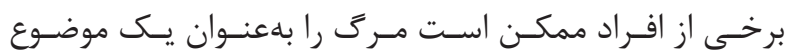

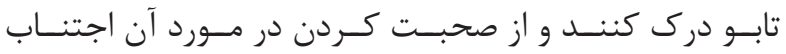

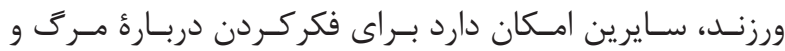

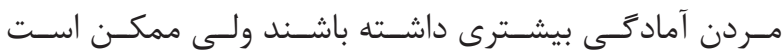

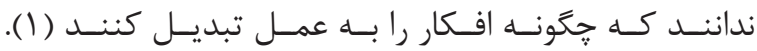

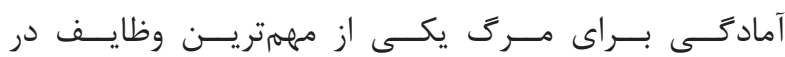

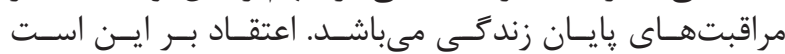

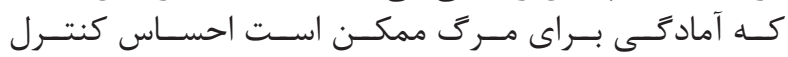

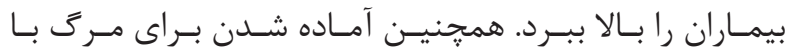

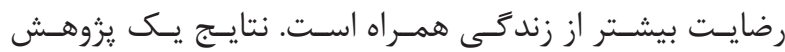

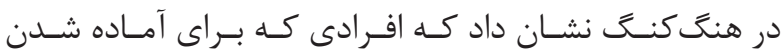

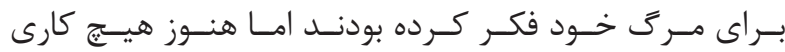

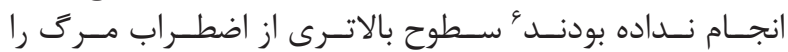

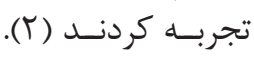

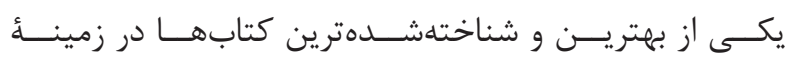

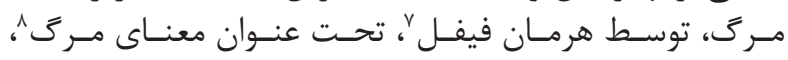

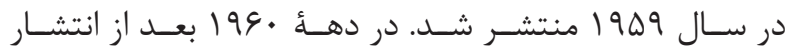

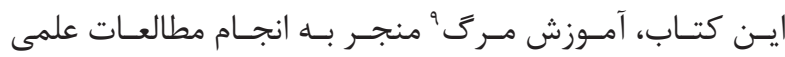

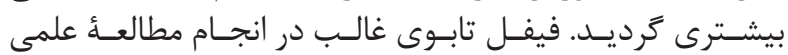

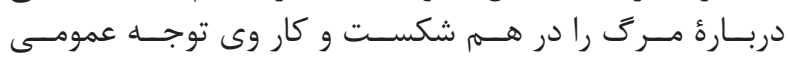

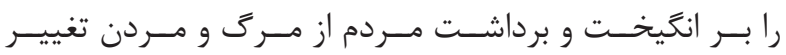

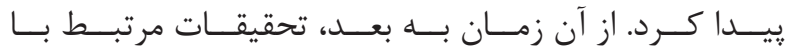

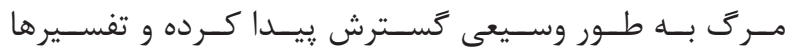

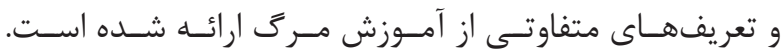

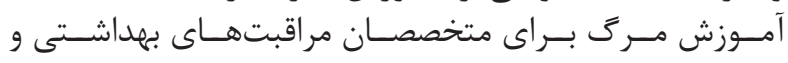

${ }^{7}$ Herman Feifel

${ }^{8}$ Meaning of death

${ }^{9}$ Death education

${ }^{10}$ Pine

${ }^{11}$ Thanatologist 


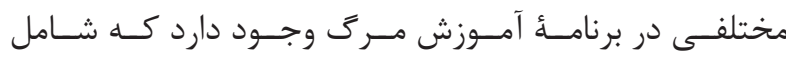

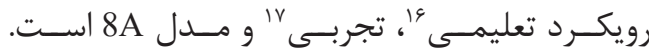

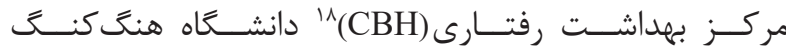

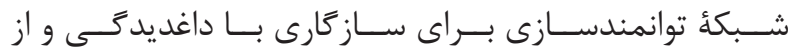

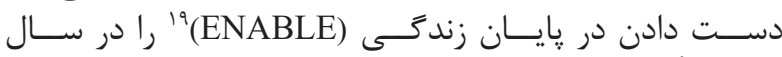

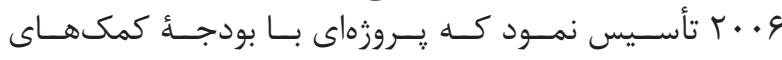

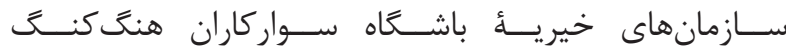

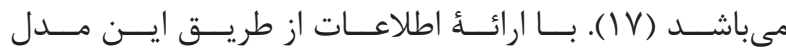

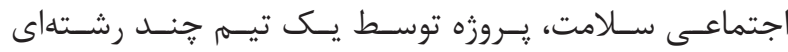

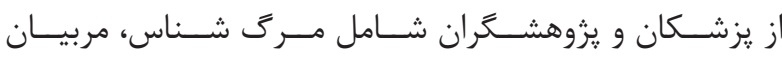

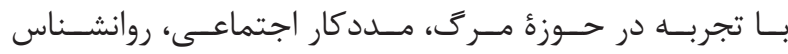

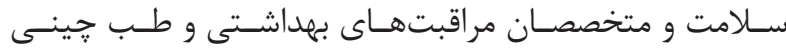

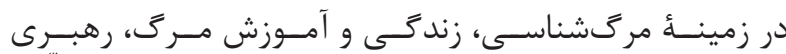

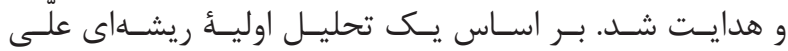

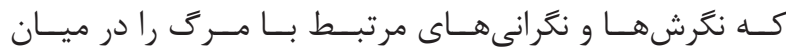

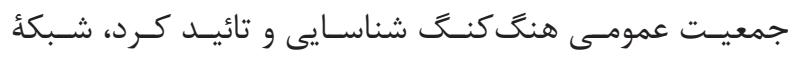

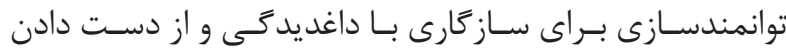

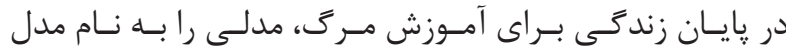

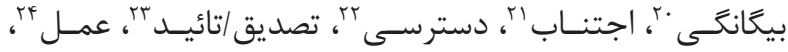

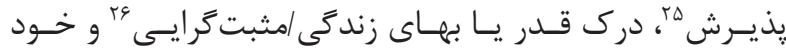

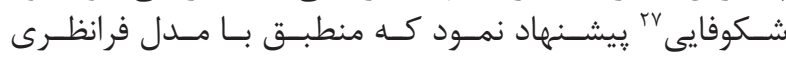

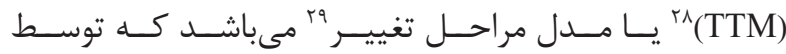

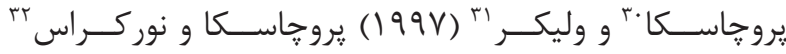

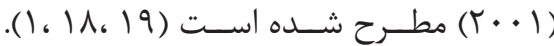

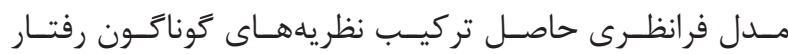

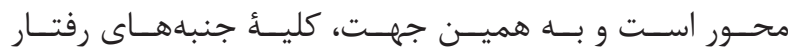

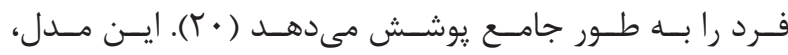

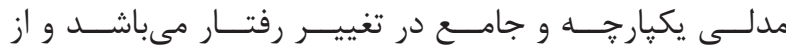

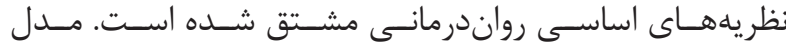

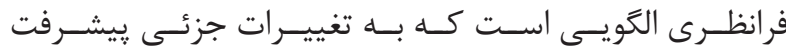

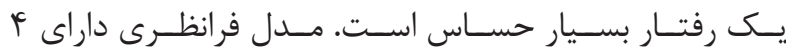

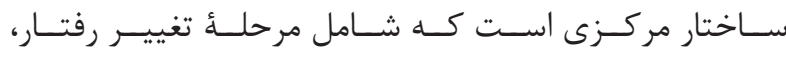

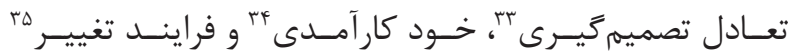

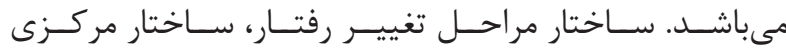

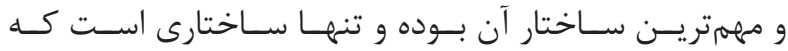

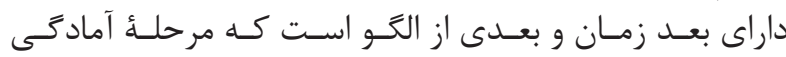

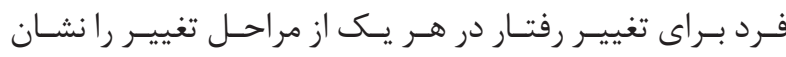

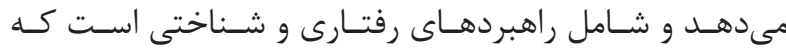

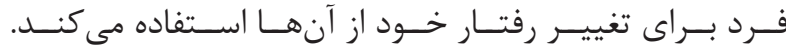

\section{${ }^{12}$ Astramovich}

${ }^{13}$ Pehrsson

${ }^{14}$ Doughty

${ }^{15}$ Hoskins

${ }^{16}$ Didactic approache

${ }^{17}$ Experiential approache

${ }^{18}$ Centre behavioral health

${ }^{19}$ Empowerment network of adjustment to bereavement and loss in end-of-life

${ }^{20}$ Alienation

${ }^{21}$ Avoidance

${ }^{22}$ Access

${ }^{23}$ Acknowledgement

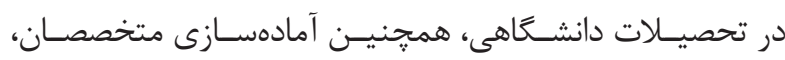

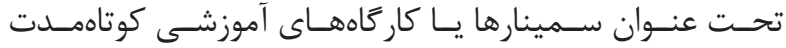

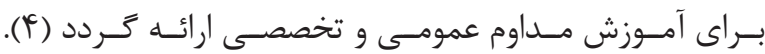

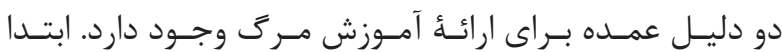

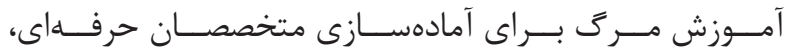

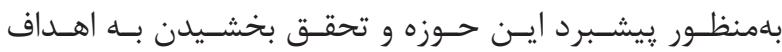

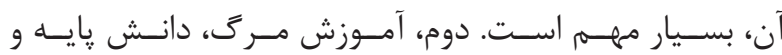

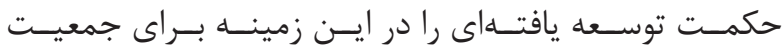

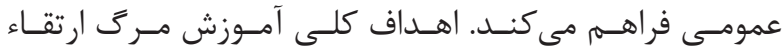

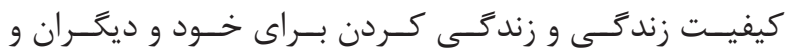

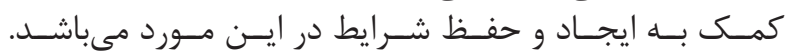

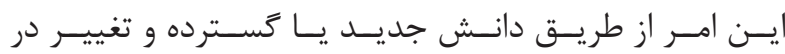

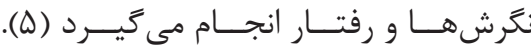

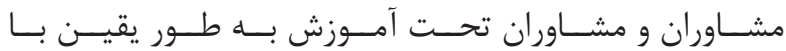

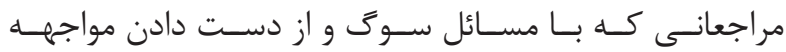

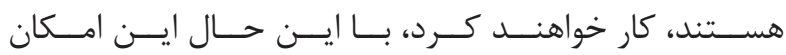

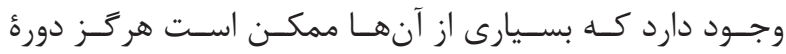

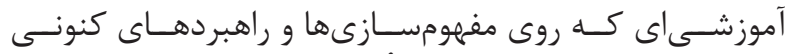

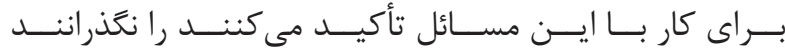

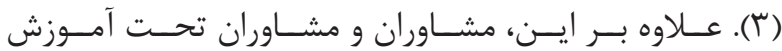

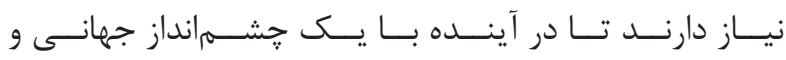

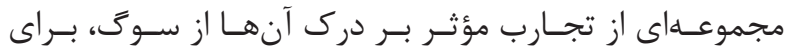

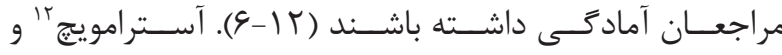

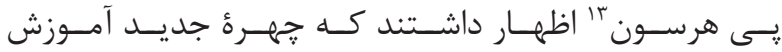

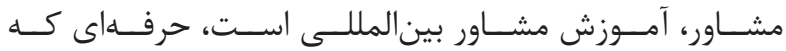

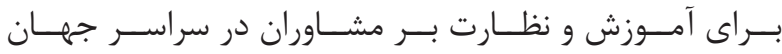

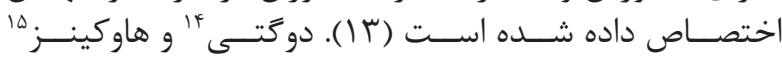

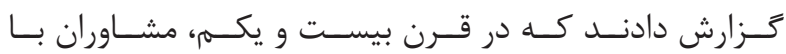

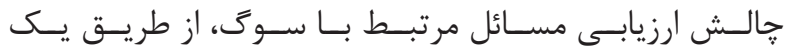

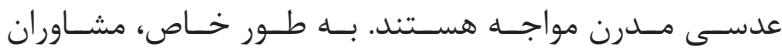

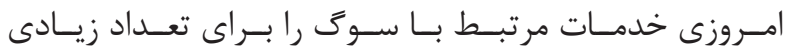

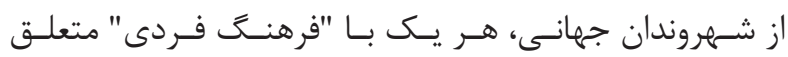

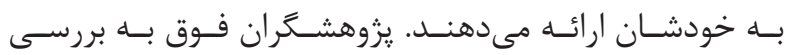

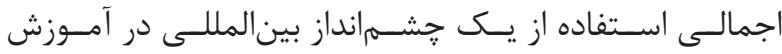

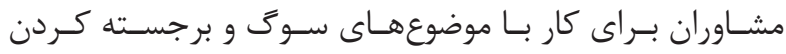

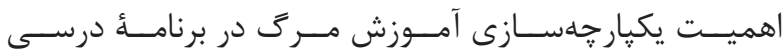

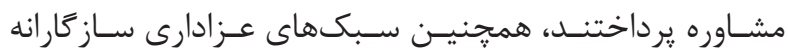

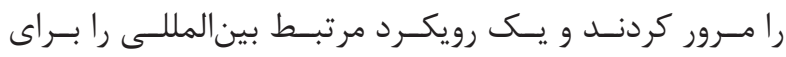

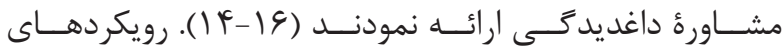
${ }^{24}$ Action
${ }^{25}$ Acceptance
${ }^{26}$ Appreciation
${ }^{27}$ Actualization
${ }^{28}$ Trans-theoretical model
${ }^{29}$ Stages of change
${ }^{30}$ Prochaska
${ }^{31}$ Velicer
${ }^{32}$ Norcross
${ }^{33}$ Decisional balance
${ }^{34}$ Self- efficacy
${ }^{35}$ Process of change 


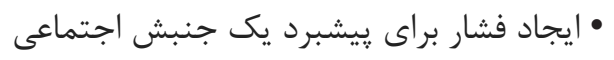

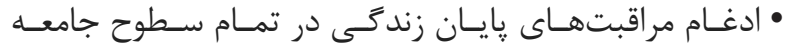

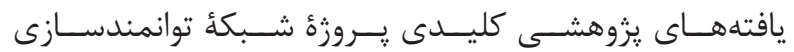

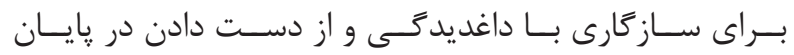

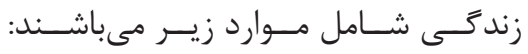

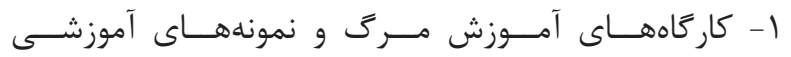

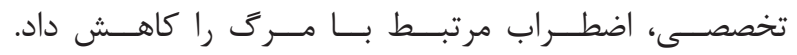

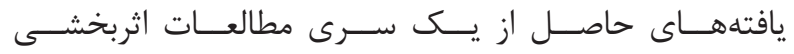

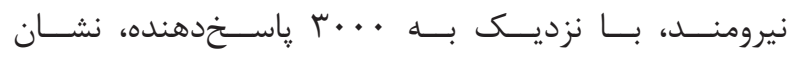

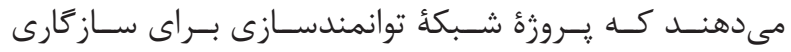

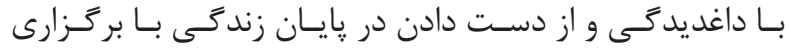

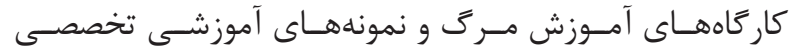

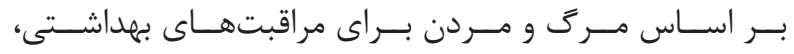

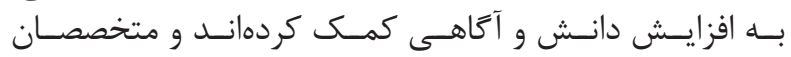

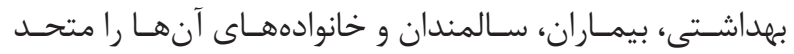

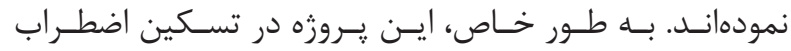

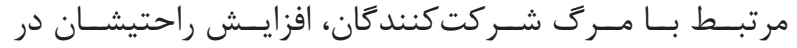

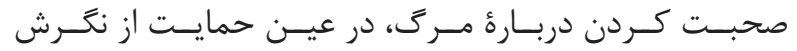

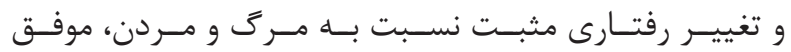
بـوده اسـت.

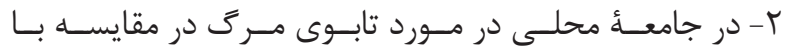

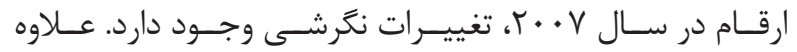

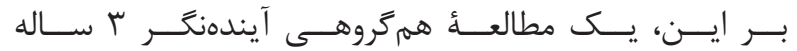

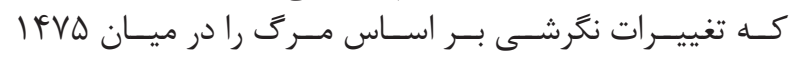

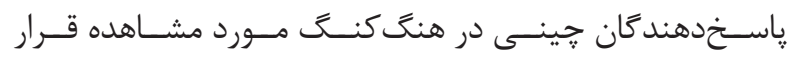

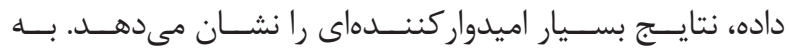

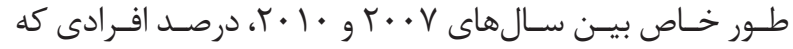

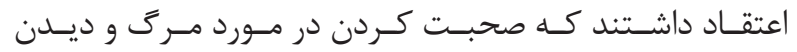

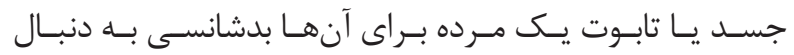

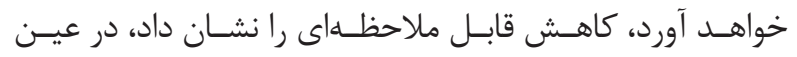

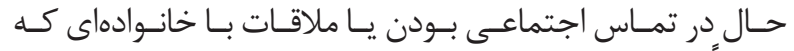

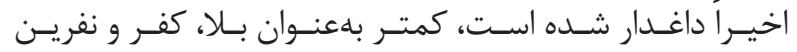

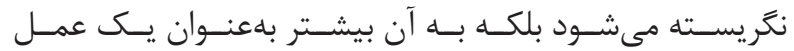

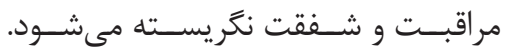

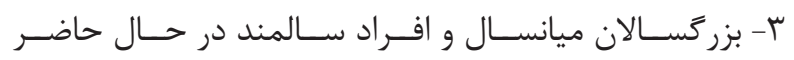

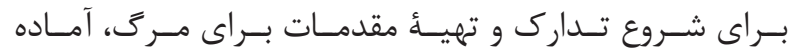

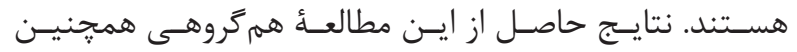

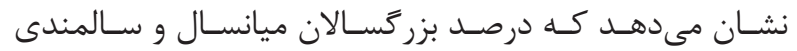

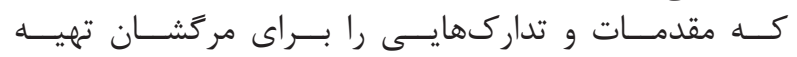

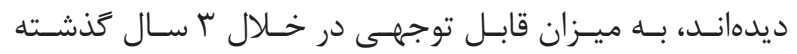

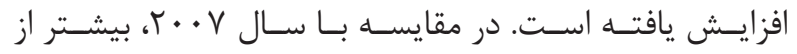

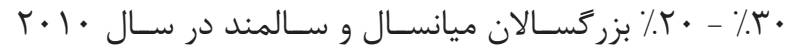

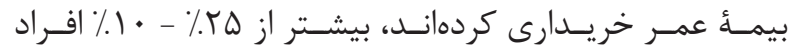

${ }^{36}$ Precontemplation

${ }^{37}$ Contemplation

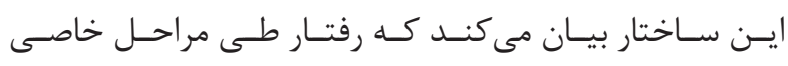

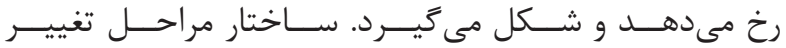

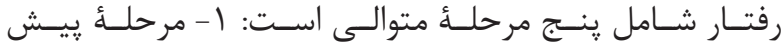

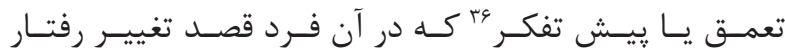

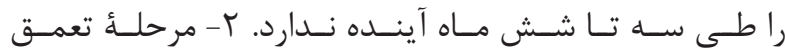

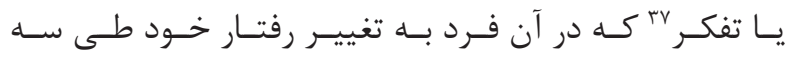

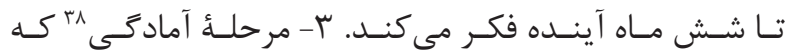

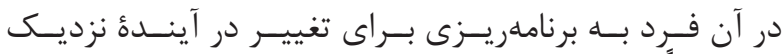

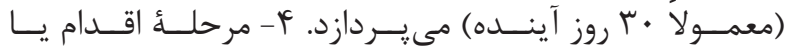

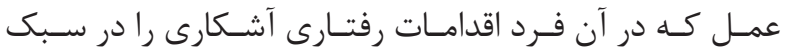

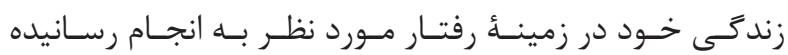

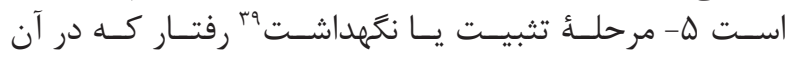

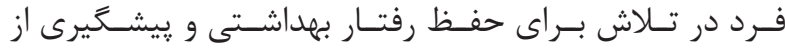

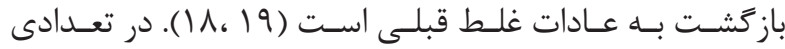

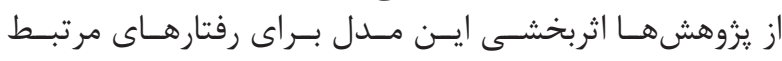

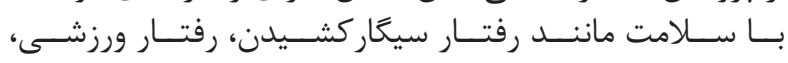

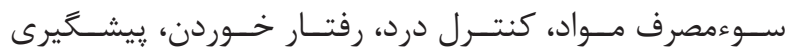

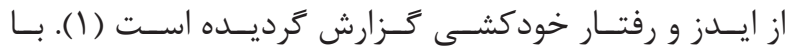

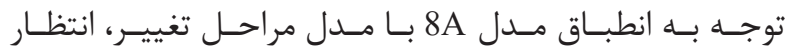

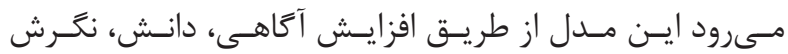

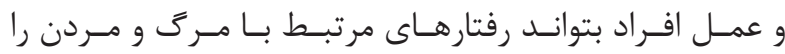

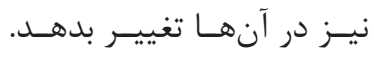

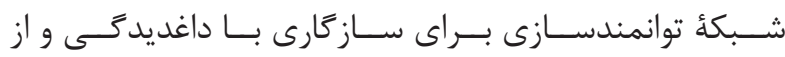

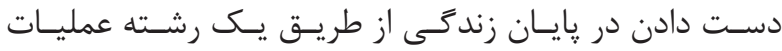

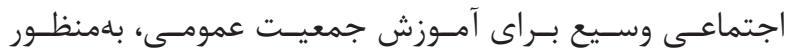

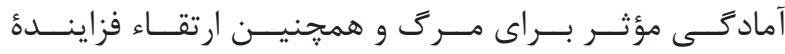

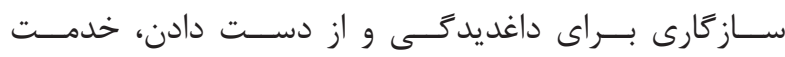

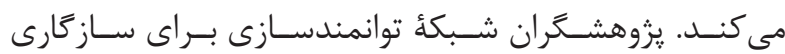

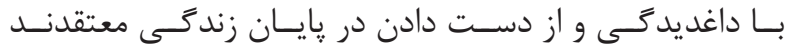

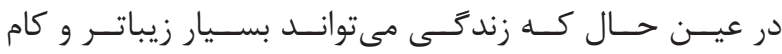

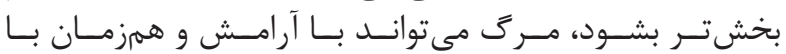

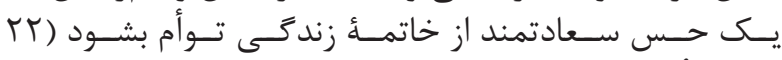

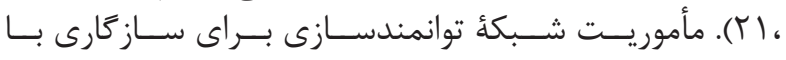

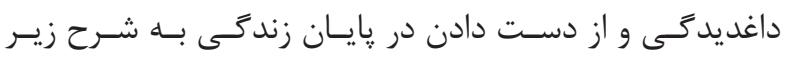
اســت

• ارتقاء آتاهى عمومى در مورد مرك، مردن و داغديدَّى

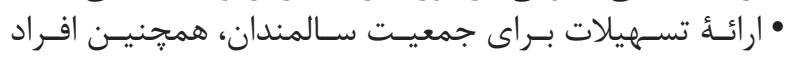

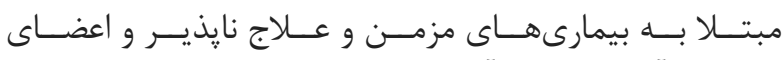

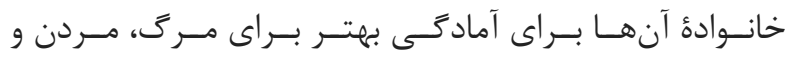

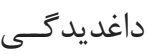

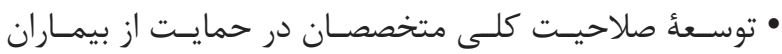

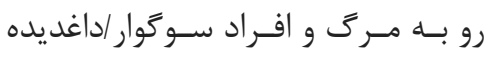

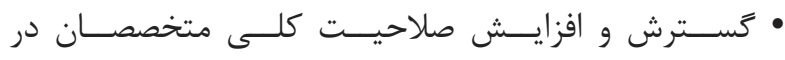

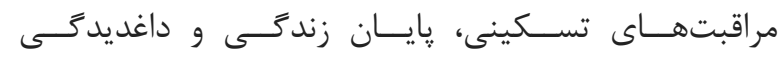

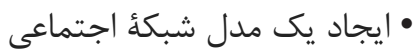

${ }^{38}$ Preparation

${ }^{39}$ Maintenance 


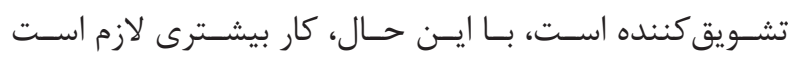

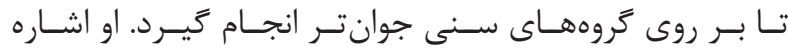

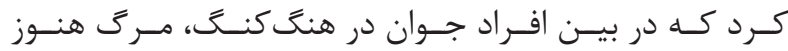

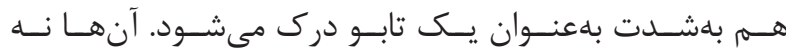

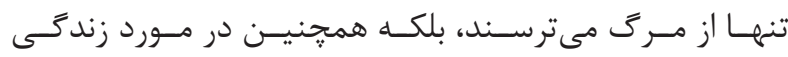

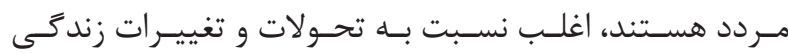

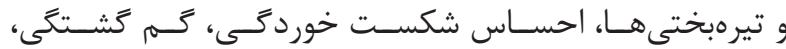

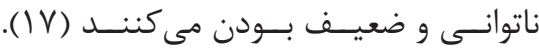

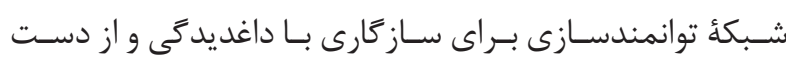

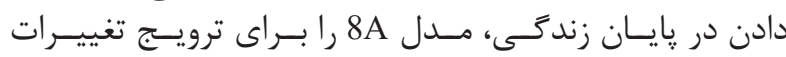

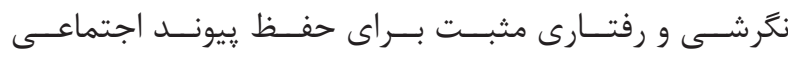

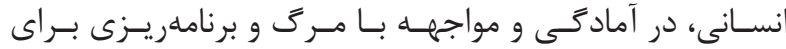

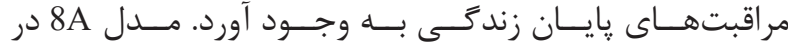

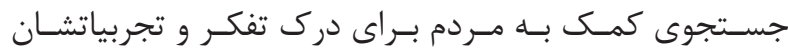

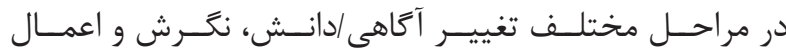

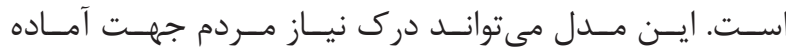

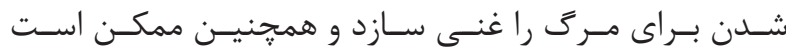

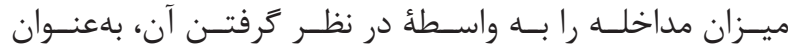

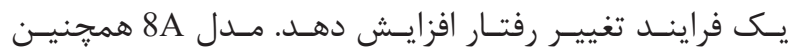

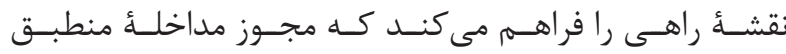

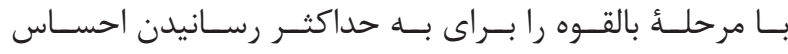

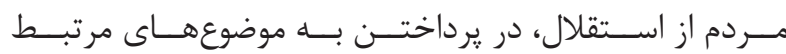

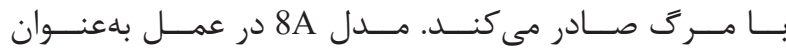

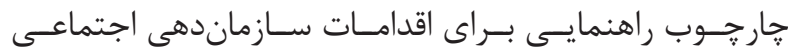

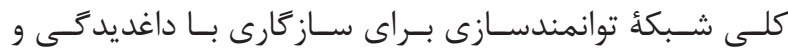

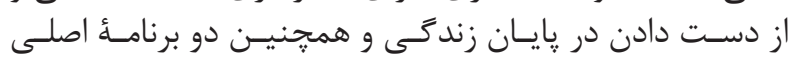

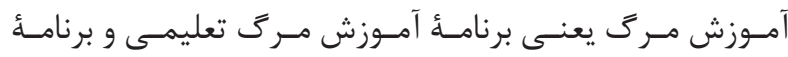

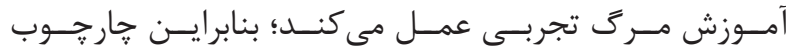

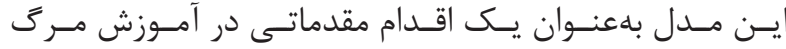

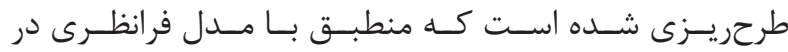

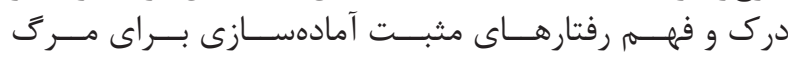

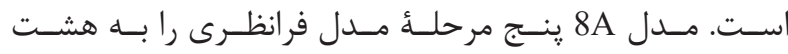

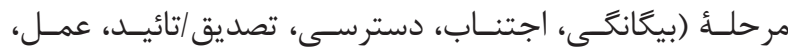

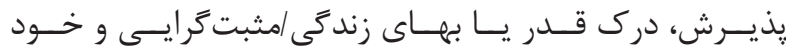

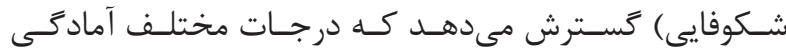

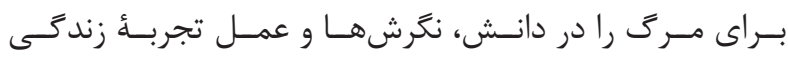

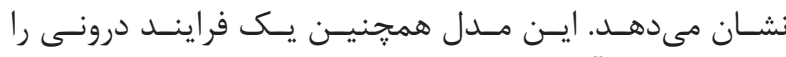

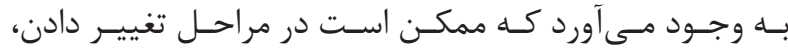

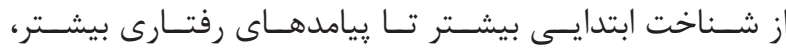

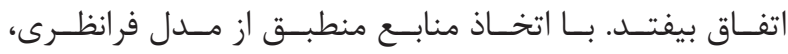

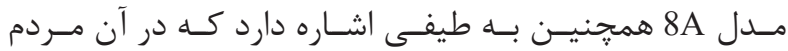

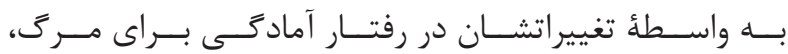

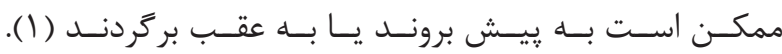

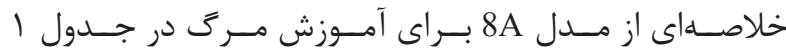

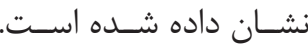

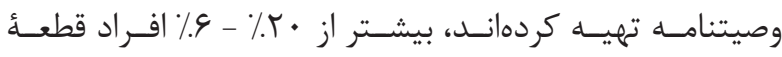

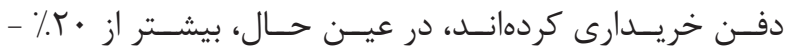

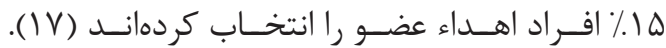

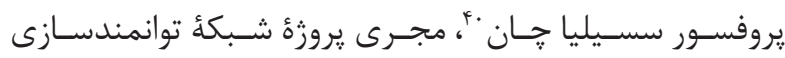

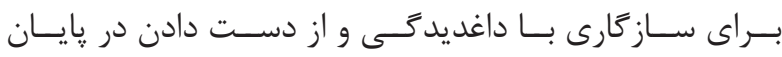

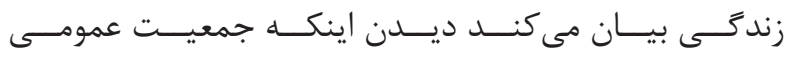

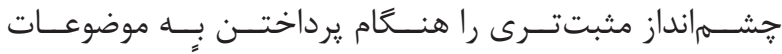

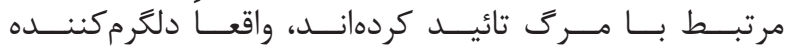

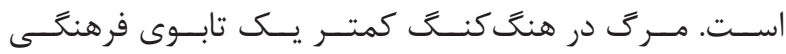

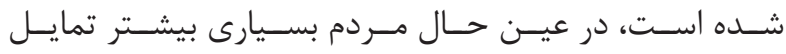

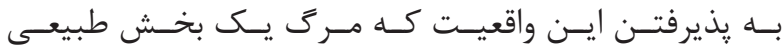

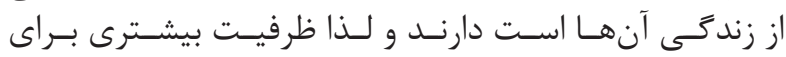

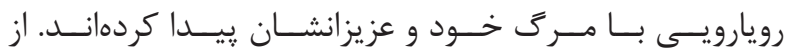

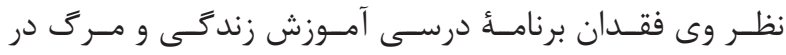

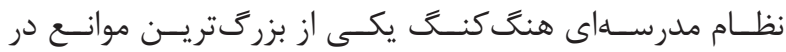

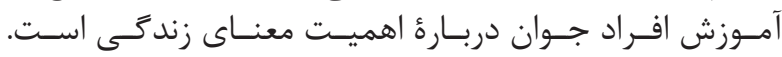

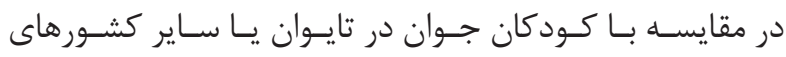

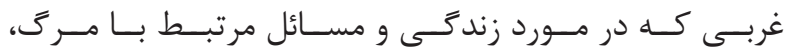

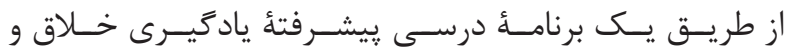

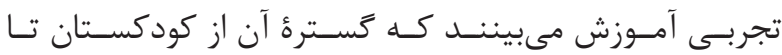

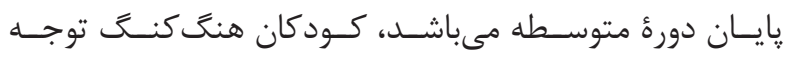

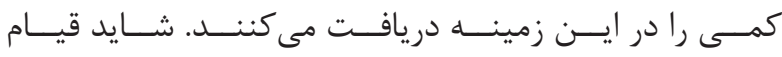

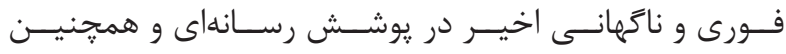

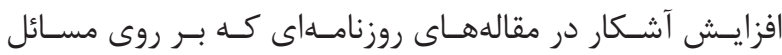

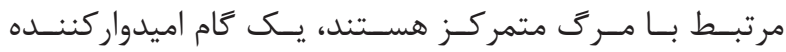

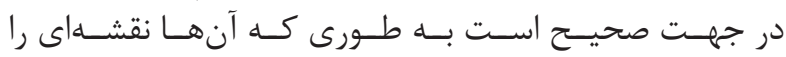

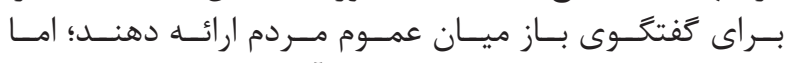

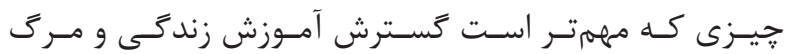

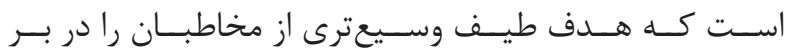

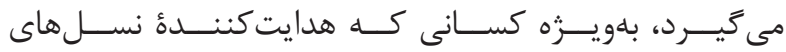

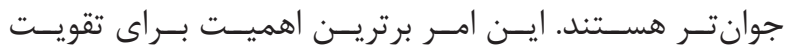

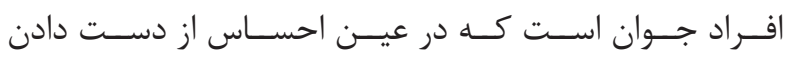

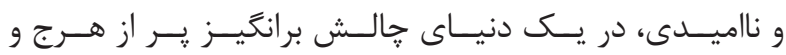

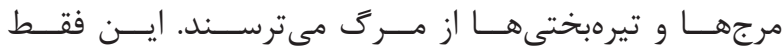

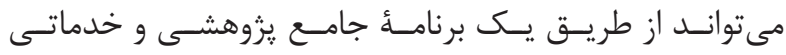

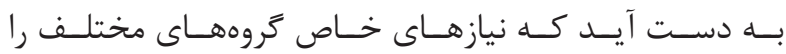

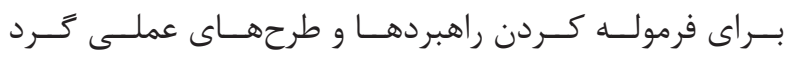

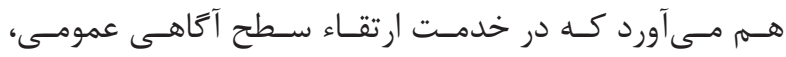

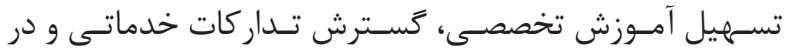

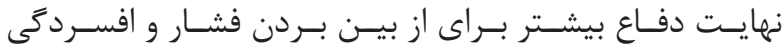

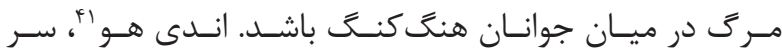

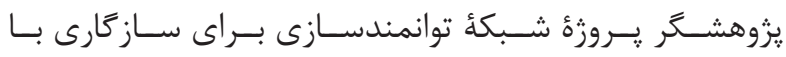

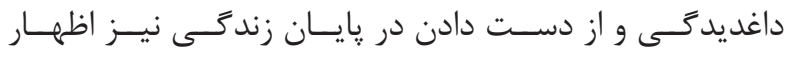

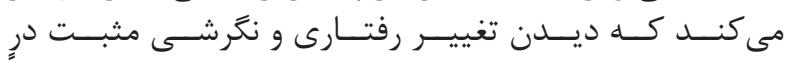

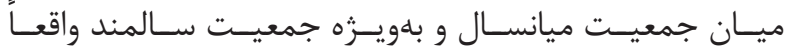

${ }^{40}$ Cecilia Chan

${ }^{41}$ Andy Hoo 
جدول ا- مدل 8A براى آموزش مركى (1).

\begin{tabular}{|c|c|c|c|}
\hline 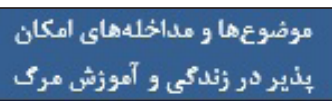 & 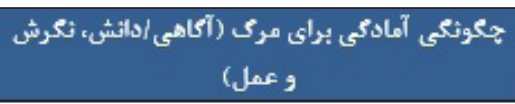 & كرايناها در ملدل 8.As & مراحل TMM \\
\hline معرفى اصطلامات و مفاهيمه متبك كريت & 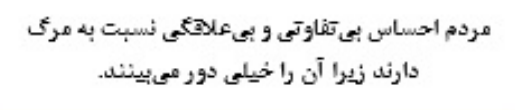 & 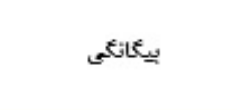 & \multirow{2}{*}{ تيشى تعمق يا تيش بيش } \\
\hline 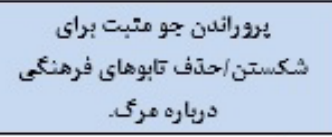 & 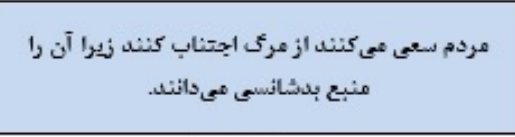 & اجتناب & \\
\hline فراهي كردن اطلاعات مربوط. & مردم به اطلاعات دربارة آمادثى مركى دسترسى نشارند. & دسترسى & تعمق يا تفكر \\
\hline فراهي كردن آموزث روائى و ارتثاء & 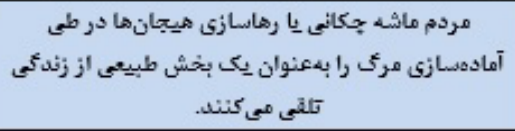 & تصديق/تاتيد/اقرار & آمادكى \\
\hline ارتقاء حس يكيارئلمى زئشيكى. & مردم مرثى را بهعنوان يكى بخش طبيعى از زئدئى تلقى & مبتيرش & \multirow{2}{*}{ اتحام/اعمل } \\
\hline حعايت از انجام طرح اتقدام. & مردم به طور فعالى مشغول برنامهريزى براى زندئى و & اقتام/م/عمل & \\
\hline 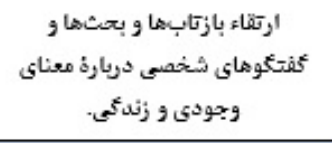 & مردم قدر زئدئى را مى داتند و معناى زئدكى را جستجو & 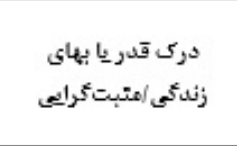 & \multirow[b]{2}{*}{ ثكهادارى و تخيير } \\
\hline 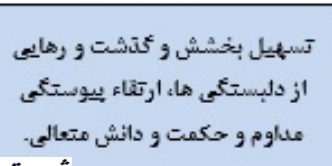 & 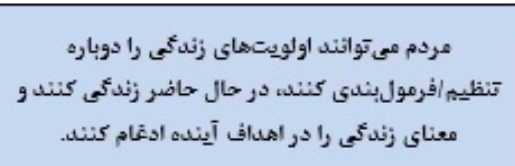 & تحقق بخشى/شكوفايع & \\
\hline
\end{tabular}

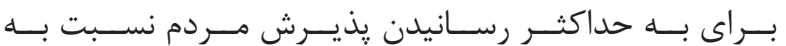

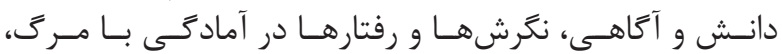

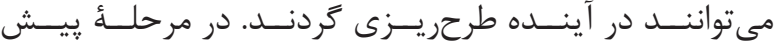

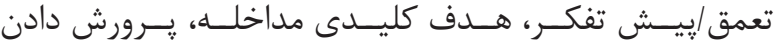

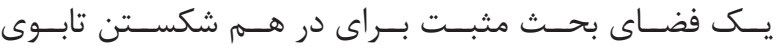

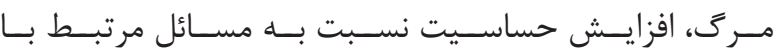

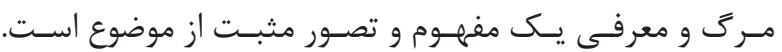

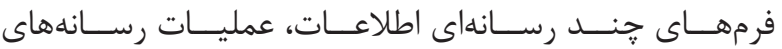

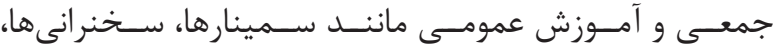

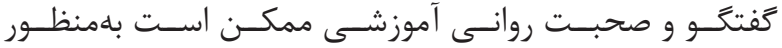

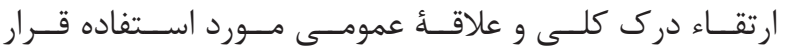

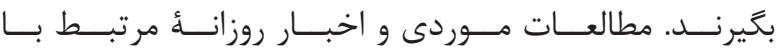

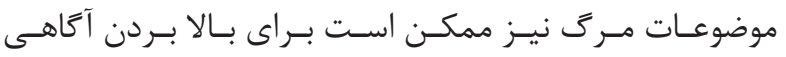

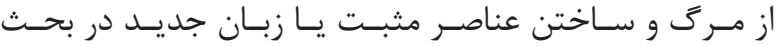

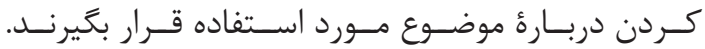
مر احل دسترسى و تصديق /تائيد

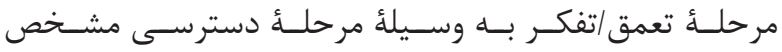

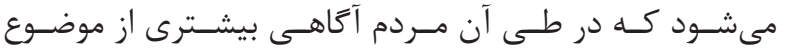

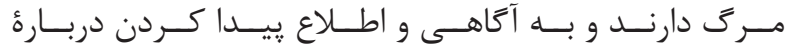

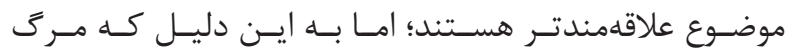

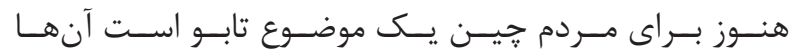

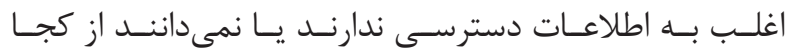

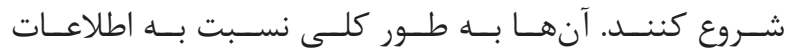

${ }^{42}$ Mental incubation

${ }^{43}$ Chow
مر احل بيعانتَى و اجتناب

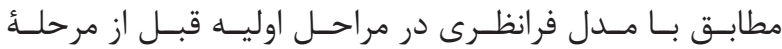

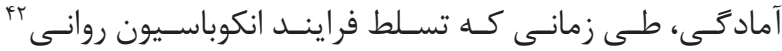

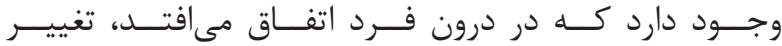

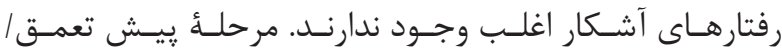

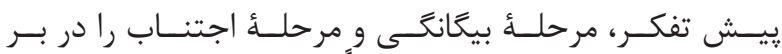

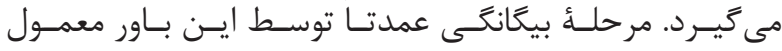

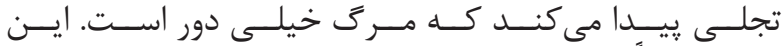

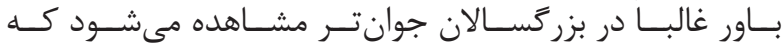

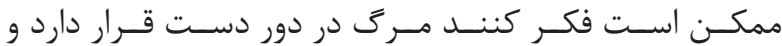

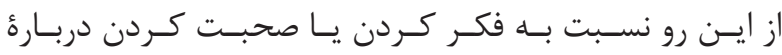

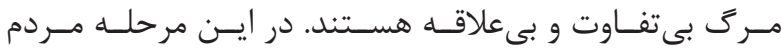

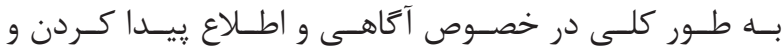

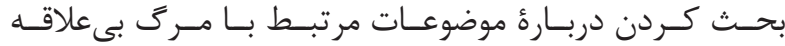

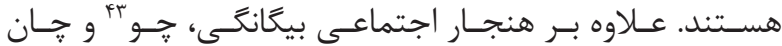

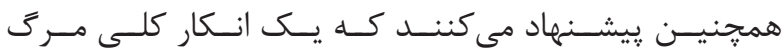

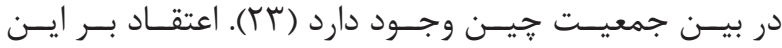

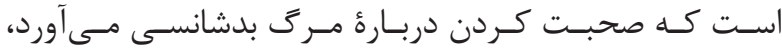

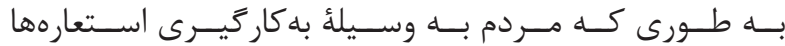

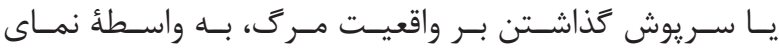

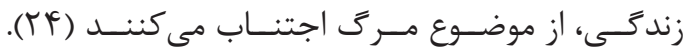

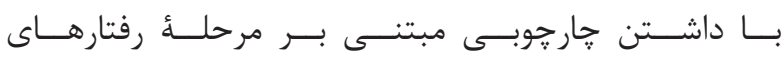

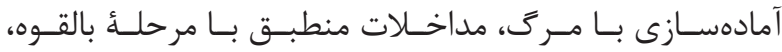




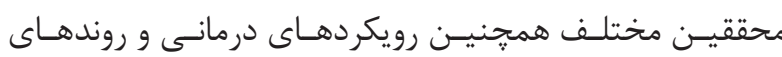

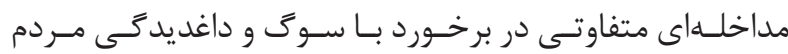

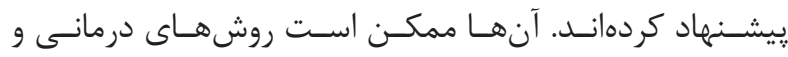

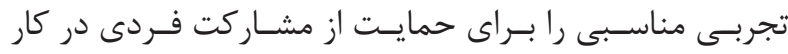

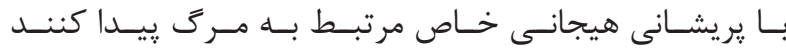

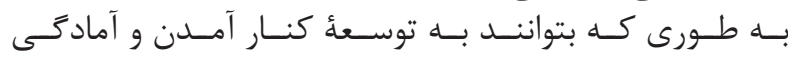

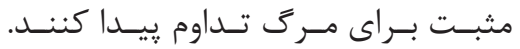

\section{مر احل هذيرش و اقدام/عمل}

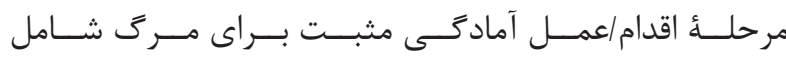

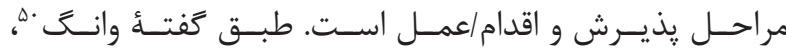

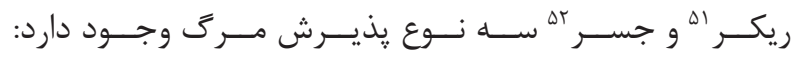

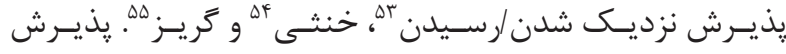

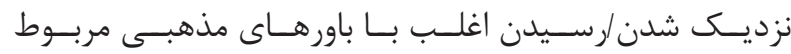

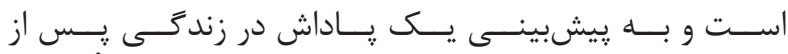

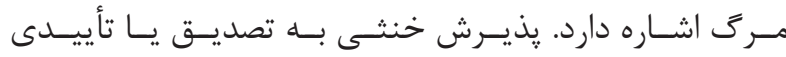

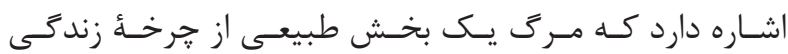

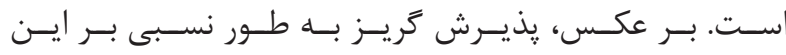

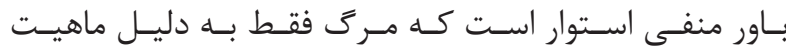

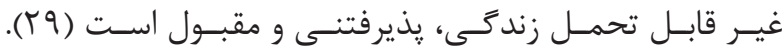

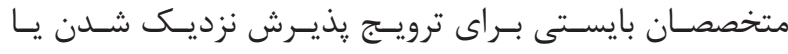

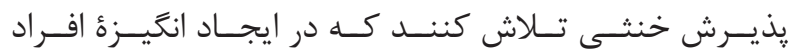

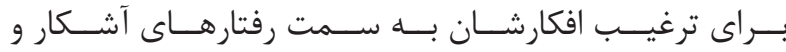

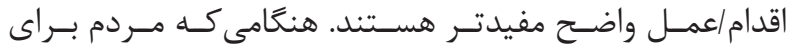

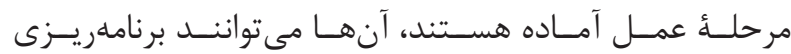

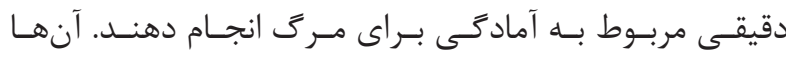

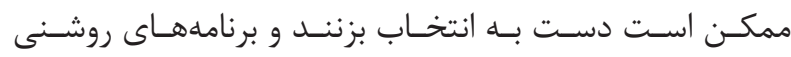

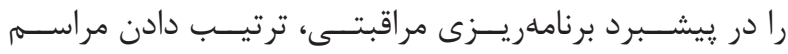

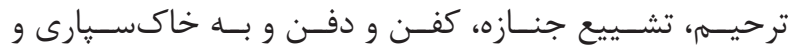

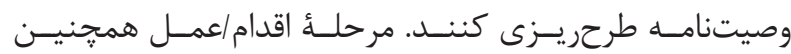

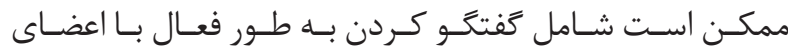

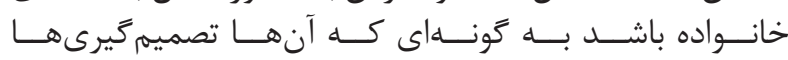

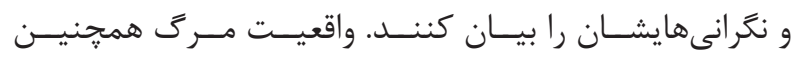

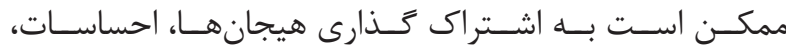

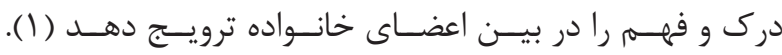

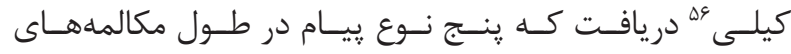

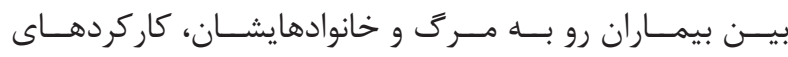

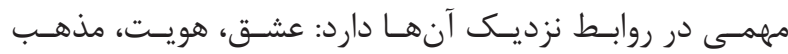

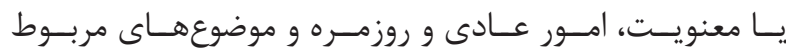

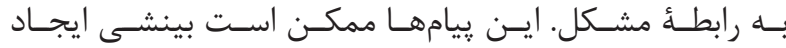

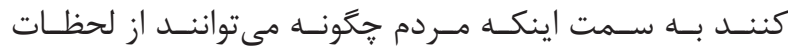

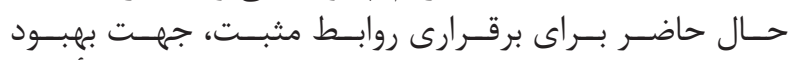

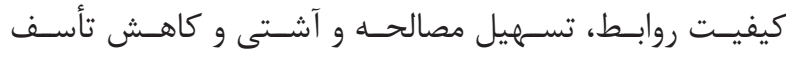

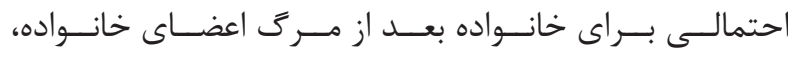

\section{${ }^{44}$ Trigger}

${ }^{45}$ Durlak

${ }^{46}$ Tomer

${ }^{47}$ Eliason

${ }^{48}$ Death anxiety model

${ }^{49}$ Existential

${ }^{50}$ Wong

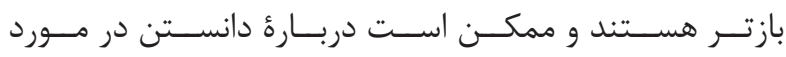

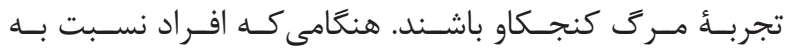

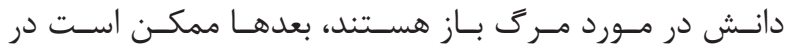

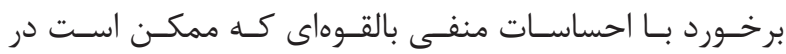

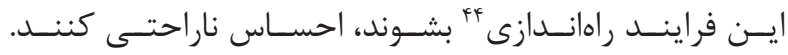

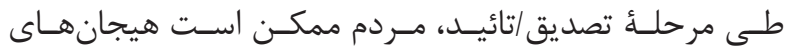

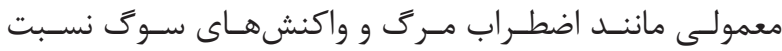

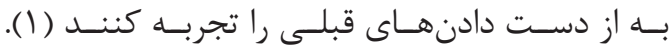

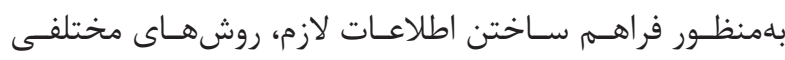

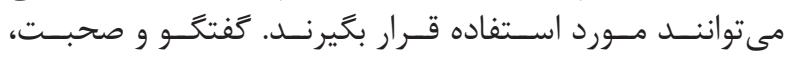

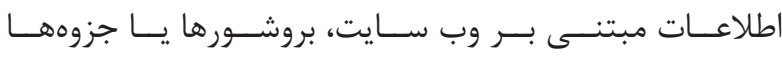

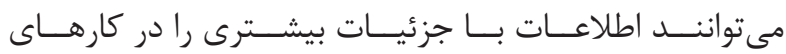

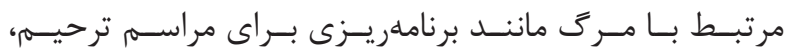

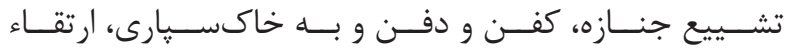

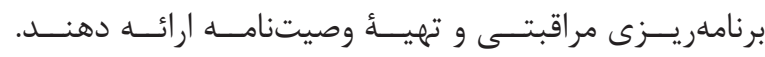

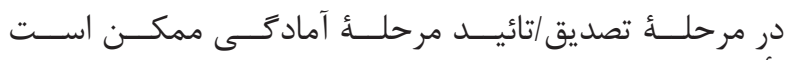

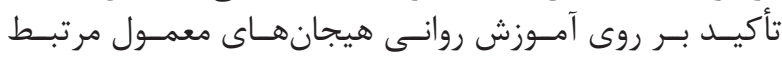

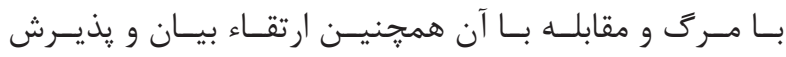

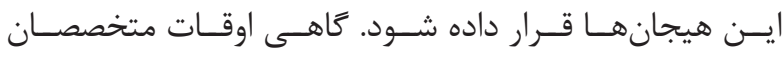

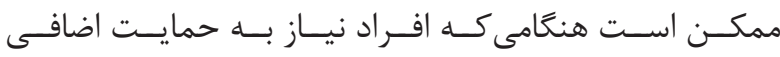

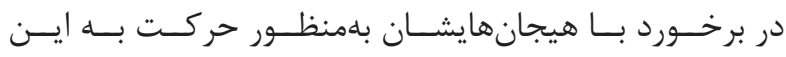

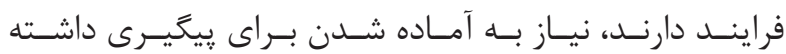

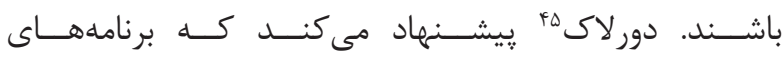

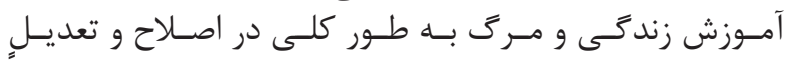

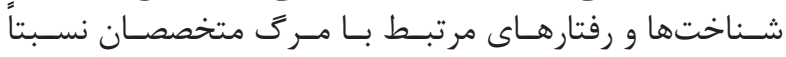

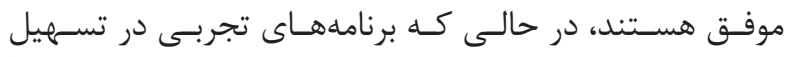

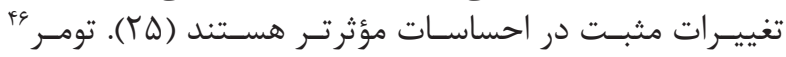

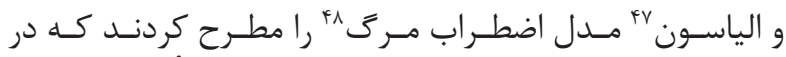

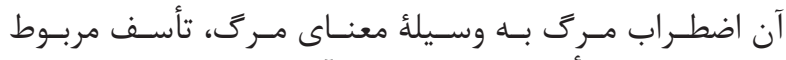

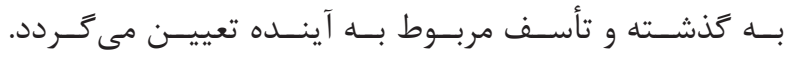

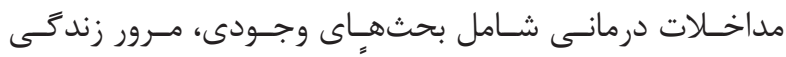

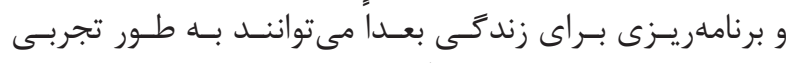

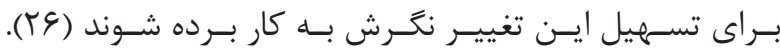

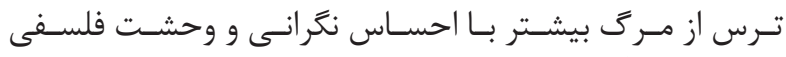

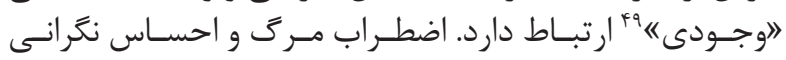

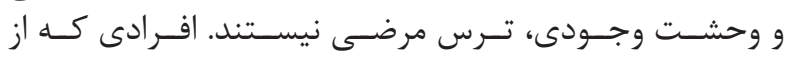

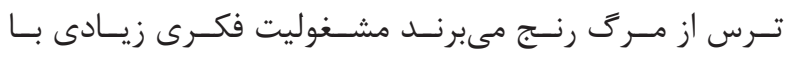

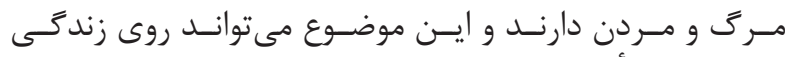

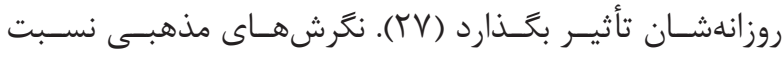

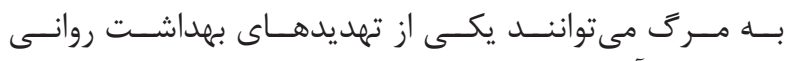

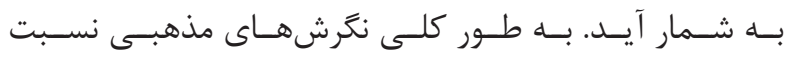

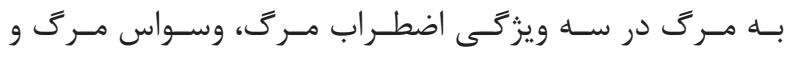

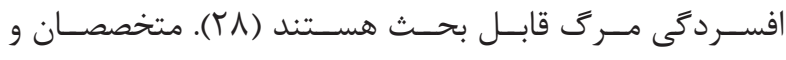
${ }^{51}$ Reker
${ }^{52}$ Gesser
${ }^{53}$ Approach
${ }^{54}$ Neutral
${ }^{55}$ Escape
${ }^{56}$ Keeley 


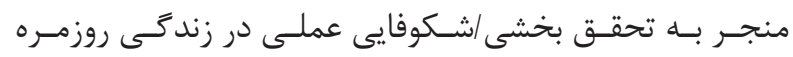

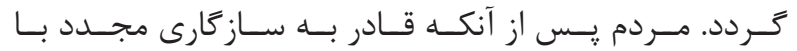

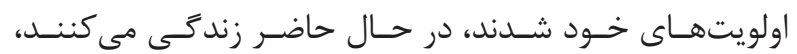

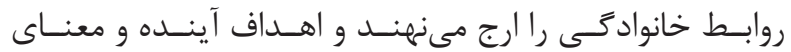

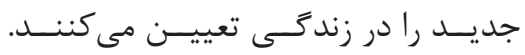

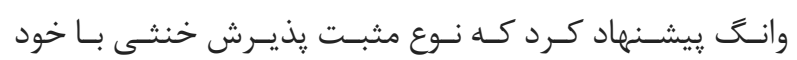

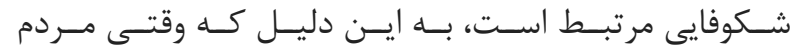

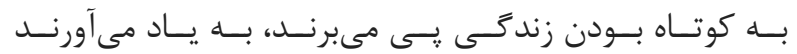

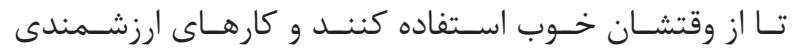

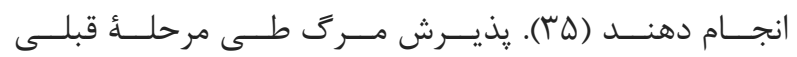

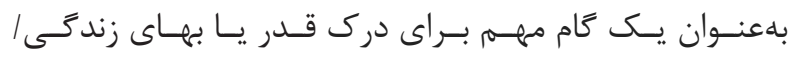

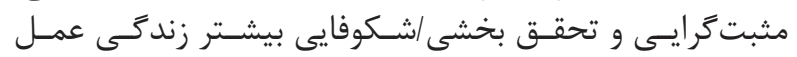

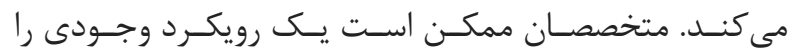

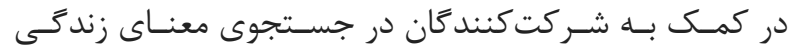

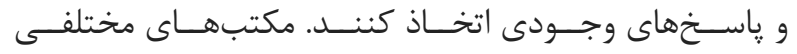

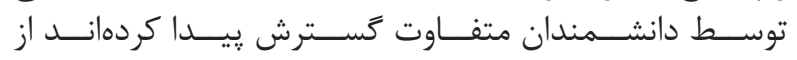

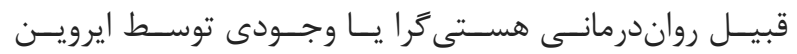

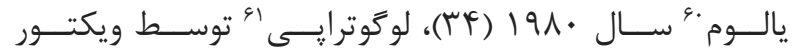

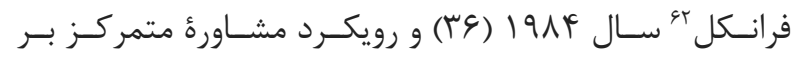

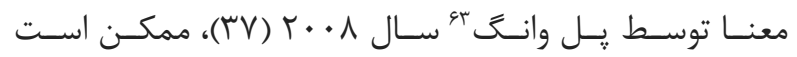

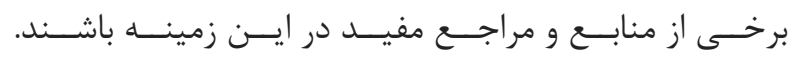

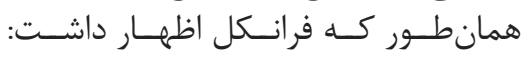

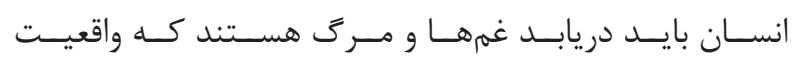

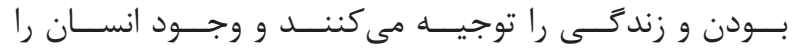

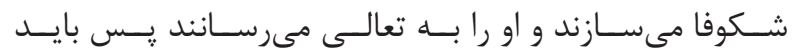

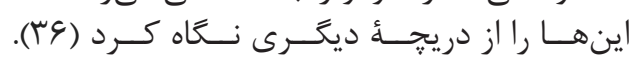

نتيجه تيرى

فوايد بالقوه مدل 8A براى آموزش مرى

بــا معرفـى مــدل 8A بــراى آمـوزش مــرى بــهـ مــدد كاران

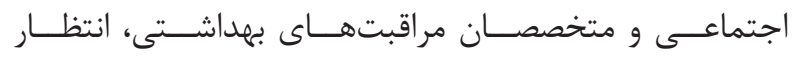

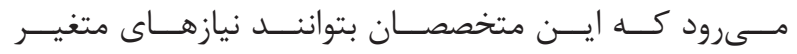

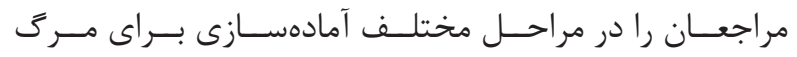

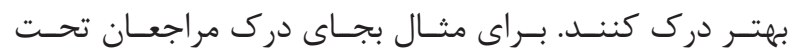

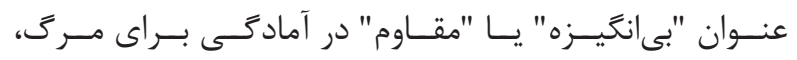

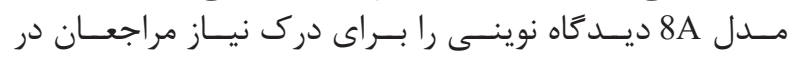

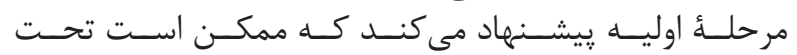

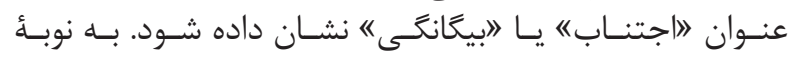

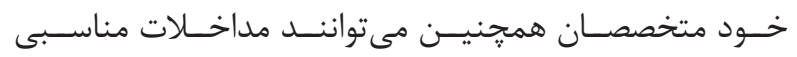

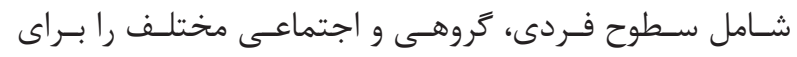

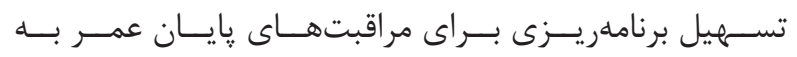

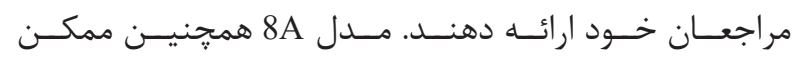

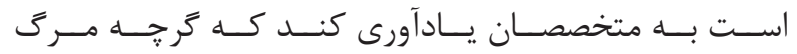

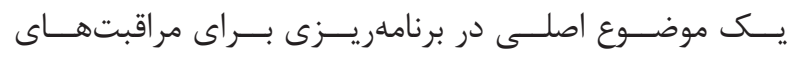

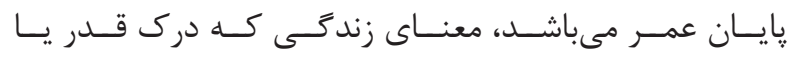

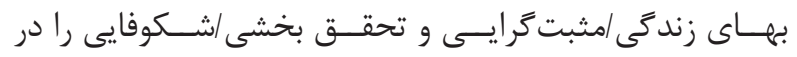

${ }^{57}$ Bulter

${ }^{58} \mathrm{Ego}$

${ }^{59}$ Boundry situation

${ }^{60}$ Irvin Yalom

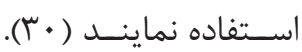

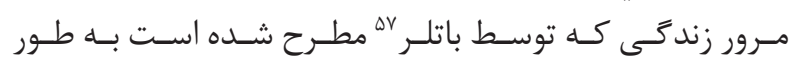

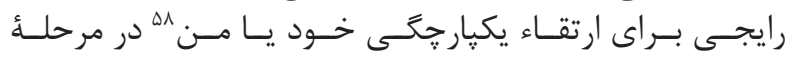

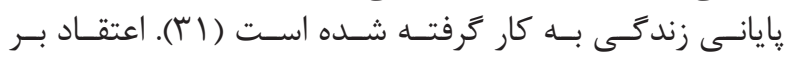

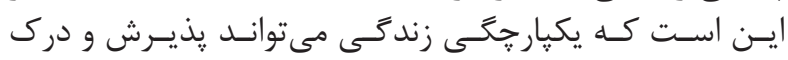

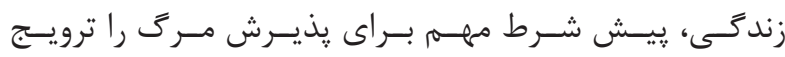

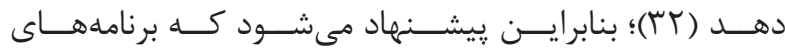

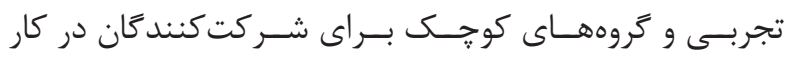

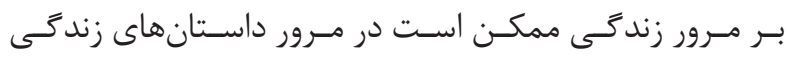

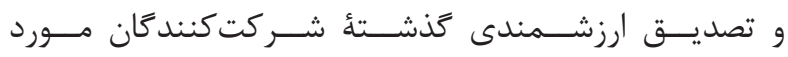

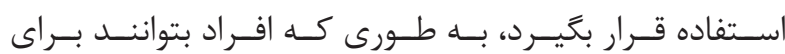

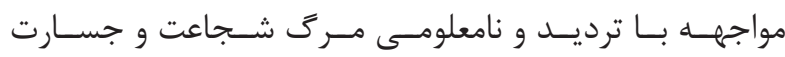

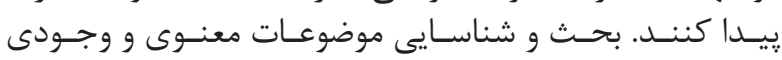

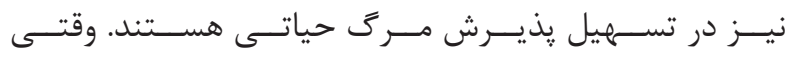

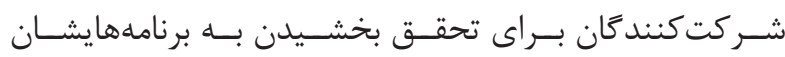

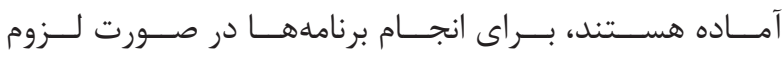

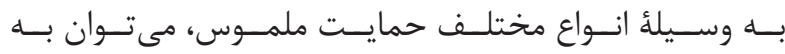

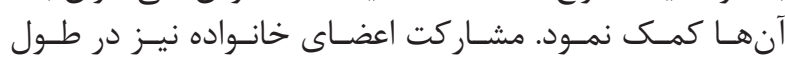

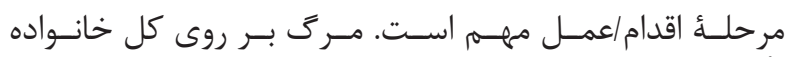

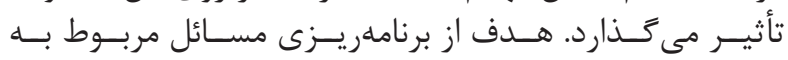

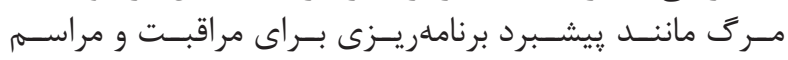

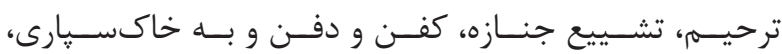

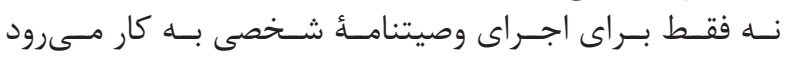

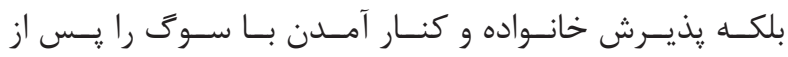

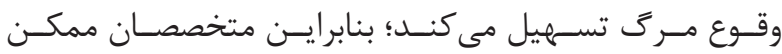

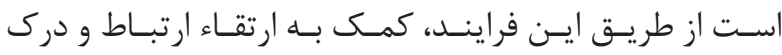

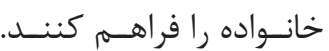

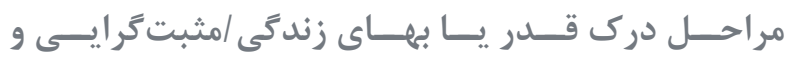

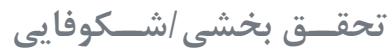

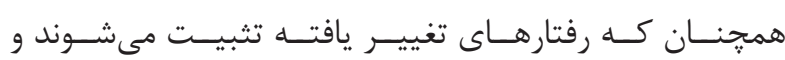

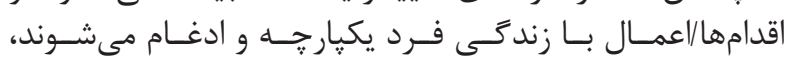

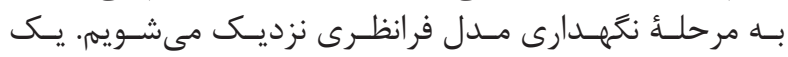

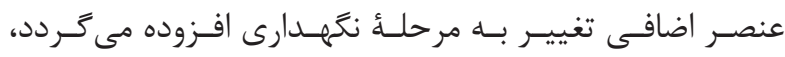

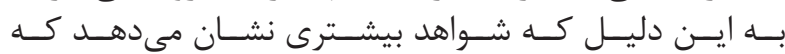

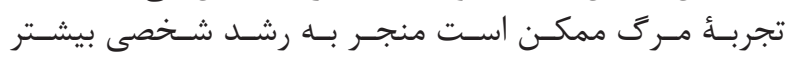

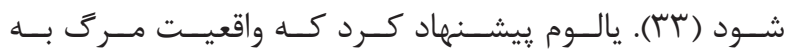

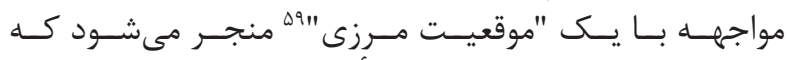

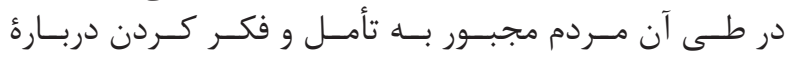

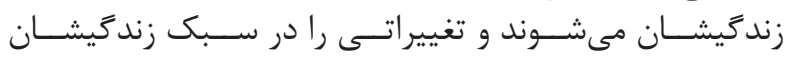

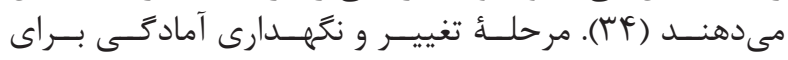

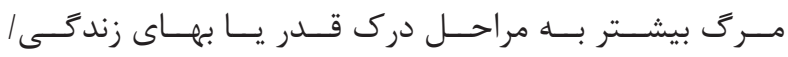

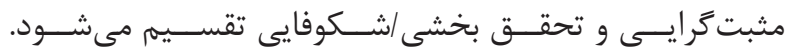

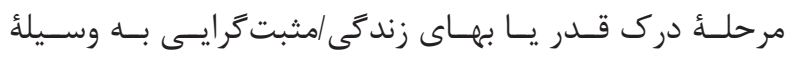

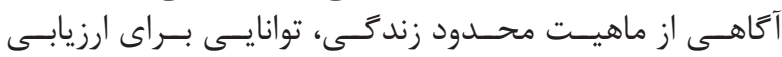

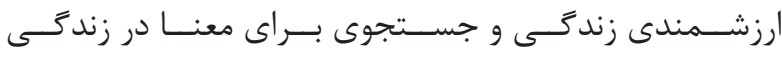

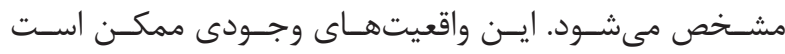

${ }^{61}$ Logotherapy

${ }^{62}$ Viktor Frankl

${ }^{63}$ Paul Wong 


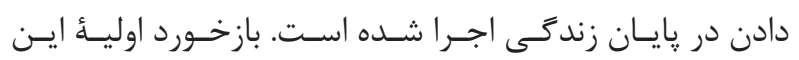

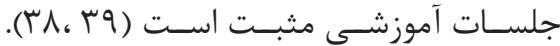

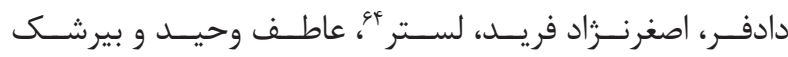

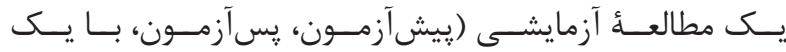

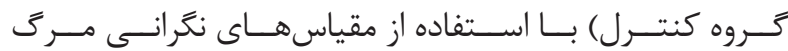
(DCS)

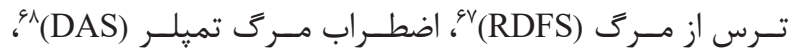

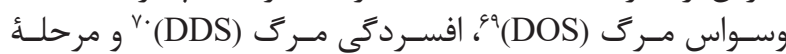

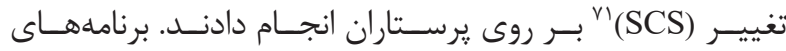

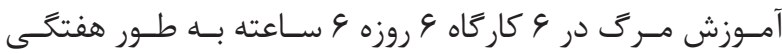

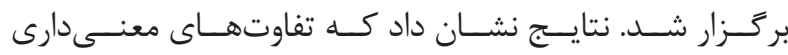

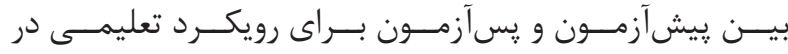

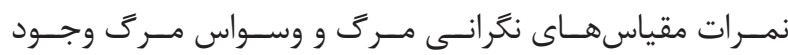

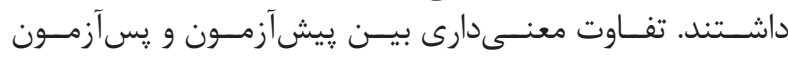

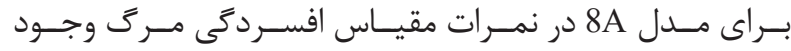

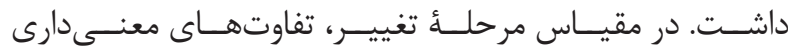

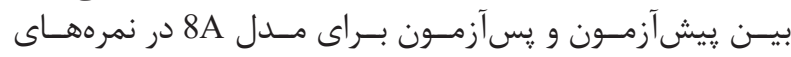

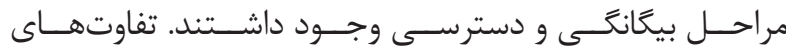

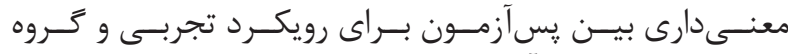

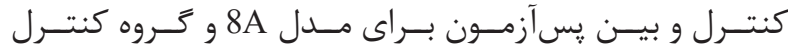

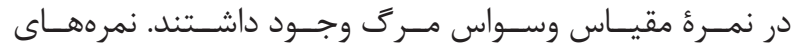

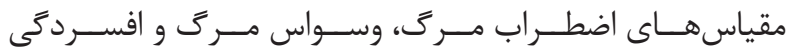

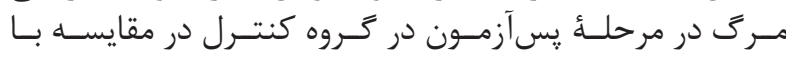

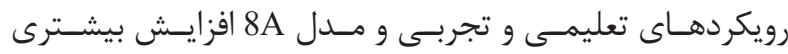

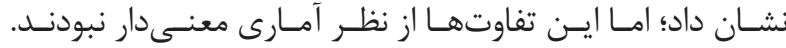

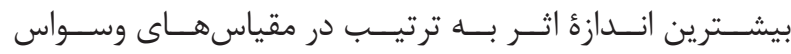

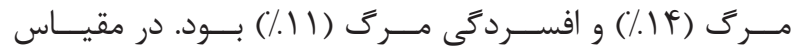

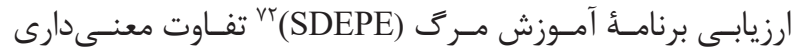

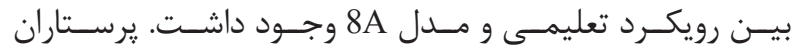

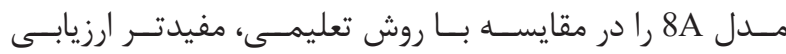

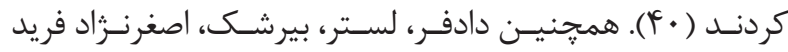

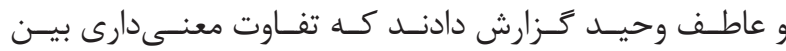

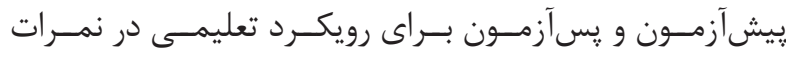

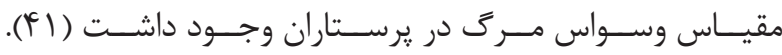

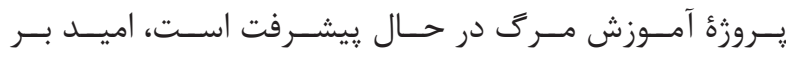

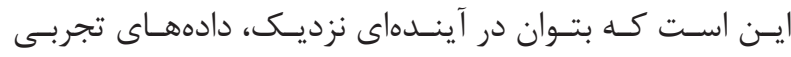

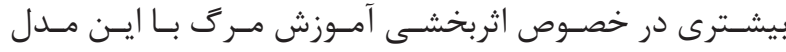

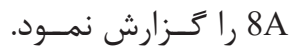

$$
\text { تشكر و قدردانى }
$$

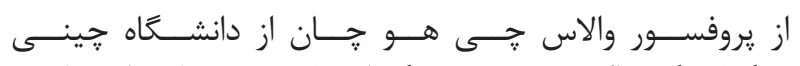

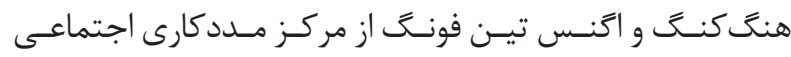

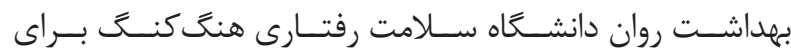

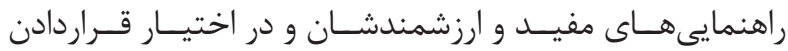

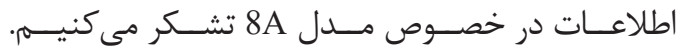

${ }^{64}$ Lester

${ }^{65}$ Death concern scale

${ }^{66}$ Collett-Lester fear of death scale

${ }^{67}$ Reasons for death fear scale

${ }^{68}$ Death anxiety scale

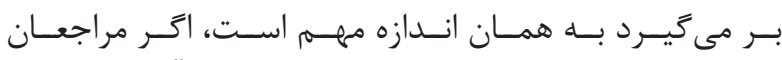

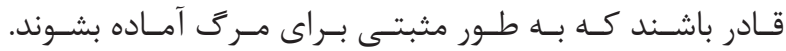

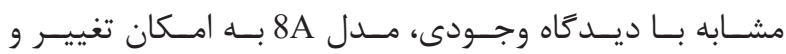

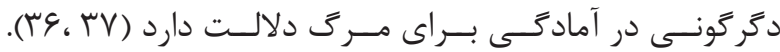

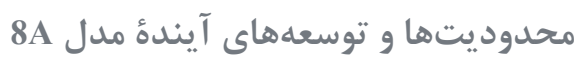

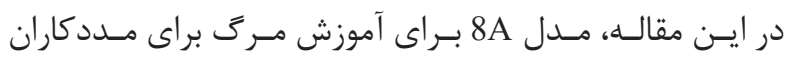

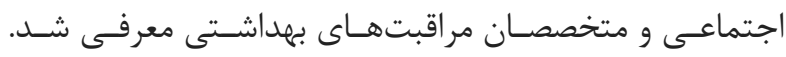

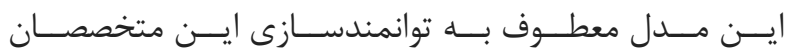

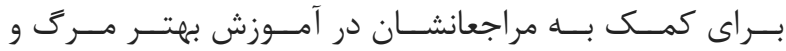

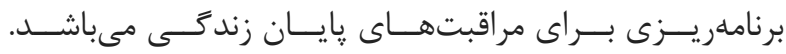

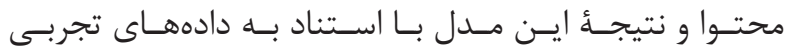

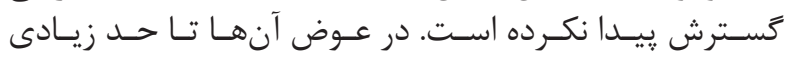

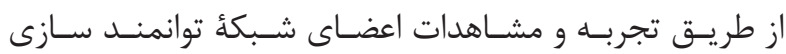

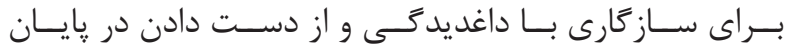

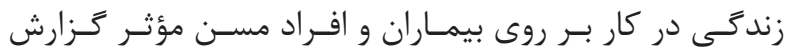

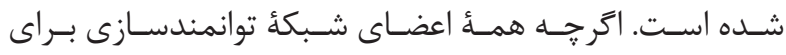

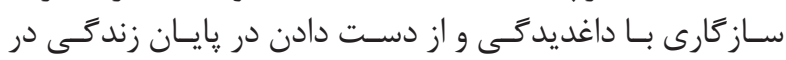

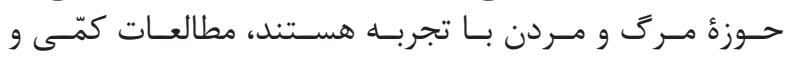

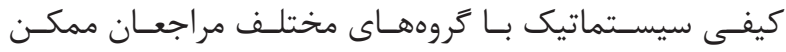

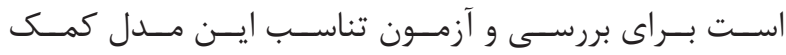

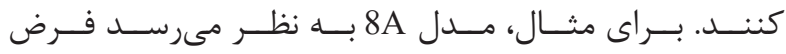

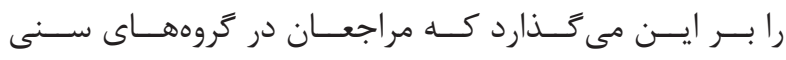

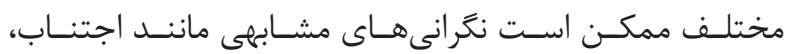

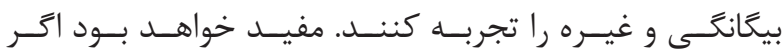

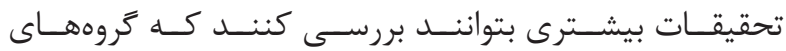

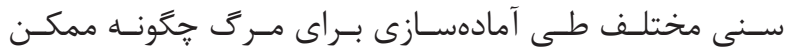

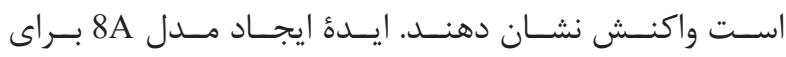

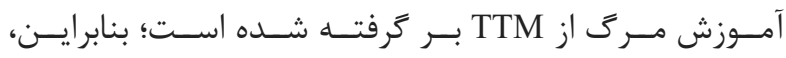

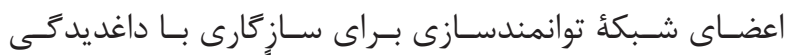

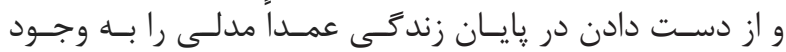

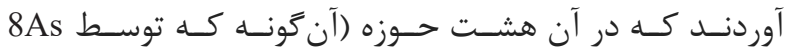

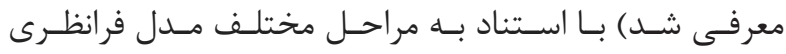

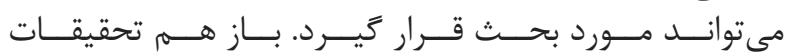

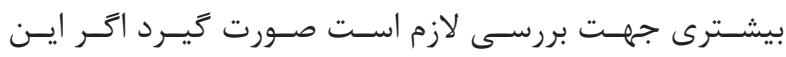

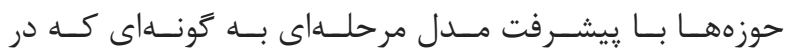

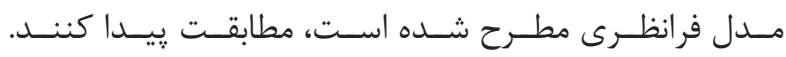

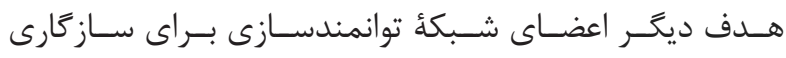

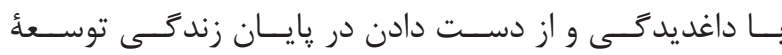

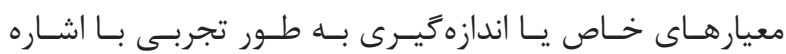

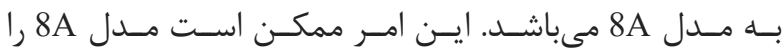

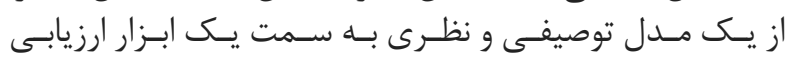

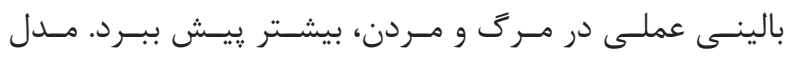

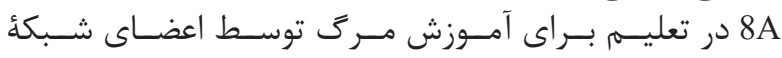

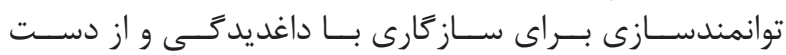

\footnotetext{
${ }^{69}$ Death obsession scale

${ }^{70}$ Death depression scale

${ }^{71}$ Stage of change scale

${ }^{72}$ Scale of death education program evaluation
} 
1. Chan WCH, Tin AF, Chan CHY, Chan CLW, Tang $\mathrm{ACW}$. Introducing the $8 \mathrm{~A}$ model in death education training: promoting planning for end - of life care for Hong Kong Chinese. Illness Crisis \& Loss. 2010; 18(1): 49-62.

2. Chan THY, Chan FM, Tin AF, Chow AY, Chan CL. Death preparation and anxiety: a survey in Hong Kong. Omega (Westport). 2006; 54(1): 67-78.

3. Wass H. Perspective on the current state of death education. Death Studies. 2004; 28(4): 289-308.

4. Moran D. Death education. www. The National Center for Death Education (NCDE). 2012.

5. Corr CA. Death education. https://en.wikipedia.org/ wiki/Death_education. 2010.

6. Harrawood LK, Doughty L, Wilde B. Death education and its effect on death anxiety in counselors-in-training. Poster Presented at Association Counselor Education and Supervision Annual Conference. San Diego, CA. 2009.

7. Harrawood LK, White LJ, Benshoff JJ. Death anxiety in a national sample of United States funeral directors and its relationship with death exposure, age, and sex. Omega (Westport). 2008; 58(2): 129-46.

8. Harrawood LK. Measuring spirituality, religiosity, and denial in individuals working in funeral service to predict death anxiety. Omega (Westport). 2009; 60(2): $129-42$.

9. Harrawood LK, Doughty L, Wilde B. Death education and its effect on death anxiety in counselors-in-training. Paper Presented at Association Counselor Education and Supervision Annual Conference. 2009b.

10. Harrawood LK, Doughty L, Wilde B. Death education and its effect on death anxiety in counselorsin-training. Paper Presented At Idaho Counseling Association Annual Conference. Couer d'Alene. 2009c.

11. Laura LK, Doughty E, Wilde B. Death education and its effect on death anxiety in counselors-intraining. Poster Presented at Kasiska College of Health Professions Research Day. Pocatello. 2009.

12. Harrawood LK, Doughty EA, Wilde B. Death education and attitudes of counselor's-in-training toward death: an exploratory study. Counseling and Values. 2011; 56: 83-95.

13. Astramovich RL, Pehrsson DE. Advancing counselor education: fostering international perspectives and open Education. 2009; 1: 1-6.

14. Horn EA, Hoskins WJ. Death education: an internationally relevant approach to grief counseling. Journal for International Counselor Education. 2011; 3 : 25-38.

15. Doughty E, Harrawood LK, Crews J. Revisiting death education: strategies for implementation. Presented rocky mountain association counselor education and supervision annual conference. Jackson: WY. 2010.

16. Doughty Horn EA, Crews JA, Harrawood LK. Grief and loss education: recommendations for curricular inclusion. Counselor Education \& Supervision. 2013; 52(1): 70-80.

17. Chan C, Hoo A. Empowerment network of adjustment to bereavement and loss in end-of-life (ENABLE). http://www.enable.hk/enabledirect. 2012.

18. Prochaska JO, Velicer WF. The transtheoretical model of health behavior change. American Journal of Health Promotion. 1997; 12(1): 38-48.

19. Prochaska JO, Norcross JC. Stages of change. Psychotherapy and Psychosomatics. 2001; 38(4): 443-8.

20. Earle S, Lioyd CE, Sidell M, Spurr S. Theory and research in promoting public health. Sociology of Health and Illness. 2008; 30(7): 1122-3.

21. Chan WC, Chan CL. Acceptance of spousal death: the factor of time in bereaved older adults' search for meaning. Death Stud. 2011; 35(2): 147-62.

22. Chan WCH, Tse HS, Chan THY. What is good death: bridging the gap between research and intervention. CLW Chan, AMY Chow. Death, dying and bereavement: a Hong Kong Chinese experience. Hong Kong: Hong Kong University Press. 2006; p. 127-35.

23. Chow AYM, Chan CLW. Introduction. CLW Chan, AYM. Chow. Death, dying and bereavement: a Hong Kong Chinese experience. Hong Kong: Hong Kong University Press. 2006b; p. 1-14.

24. Koo BWS, Tin AF, Koo EWK, Lee SM. When east meets west: implications for bereavement counseling. CLW Chan, AYM Chow. Death, dying and bereavement: a Hong Kong Chinese experience. Hong Kong: Hong Kong University Press. 2006; p. 261-71.

25. Durlak JA. Changing death attitudes through death education. RA Neimeyer. Death anxiety handbook: Research, instrumentation and application. London and 
New York: Routledge. 1994; p. 243-60.

26. Eliason G. Spirituality and counseling of the older adult. Tomer A. Death attitudes and the older adults, theories, concepts and applications. Brunner-Routledge. 2000; p. 241-54.

27. Fritscher L. "Thanatophobia: fear of death". http:// phobias.about.com/od/phobiaslist/a/thanatophobia.htm.

28. Abdel-Khalek AM. The death distress donstruct and scale. Omega (Westport). 2012; 64(2): 171-84.

29. Wong PTP, Reker GT, Gesser G. Death attitude profile-revised: a multidimensional measure of attitudes toward death. RA Neimeyer. Death anxiety handbook: research, instrumentation and application. London and New York: Routledge. 1994; p. 121-48.

30. Keeley MP. Turning toward death together: the functions of messages during final conversations in close relationships. Journal of Social and Personal Relationships. 2007; 24(2): 225-53.

31. Bulter RN. Age, death and life review. KJ Doka. Living with grief: loss in later life. Washington, DC: Hospice Foundation of America. 2002; p. 3-12.

32. Erikson E. Childhood and society. 2nd ed. New York: Norton; 1963.

33. Calhoun LG, Tedeschi RG. Posttraumatic growth: the positive lessons of loss. RA Neimeyer. Meaning reconstruction and the experience of loss. Washington, DC: American Psychological Association. 2001; p. 157-72.
34. Yalom ID. Existential psychotherapy. New York: Basic Books. 1980.

35. Wong PTP. Meaning of life and meaning of death in successful aging. a Tomer. Death attitudes and the older adult: theories, concepts, and applications. Routledge. 2000; p. 23-36.

36. Frankl V. Man's search for meaning. New York: Pocket Books. 1984.

37. Wong PTP. Meaning management theory and death acceptance. a Tomer, GT Eliason, PTP Wong. Existential and spiritual issues in death attitudes. New York/London: Lawrence Erlbaum Associates. 2008; p. 65-90.

38. Dadfar M, Lester D. Death education program: a practical guide for healthcare professionals. Mir-Mah Publications. 2014.

39. Dadfar M, Lester D, Atef Vahid MK. Asgharnizad Farid AA, Birashk B. Death distress in nurses: psychoeducational interventions. Mir-Mah Publications. 2015.

40. Dadfar M, Asgharnejad Farid AA, Lester D, Atef Vahid MK, Birashk B. Effectiveness of death education program by methods of didactic, experimental, and $8 \mathrm{~A}$ model on the reduction of death distress among nurses. International Journal of Medical Research \& Health Sciences (IJMRHS). 2016; 5(7): 60-71.

41. Dadfar M, Lester D, Birashk B, Asgharnejad Farid AA, Atef Vahid MK. The effectiveness of didactic approach on the reduction of death obsession. Shefaye Khatam. 2016; 4(S1): 29. 